\title{
Phenomenological relations for axial quasinormal modes of neutron stars with realistic equations of state
}

\author{
J. L. Blázquez-Salcedo, ${ }^{1}$ L. M. González-Romero, ${ }^{1}$ and F. Navarro-Lérida ${ }^{2}$ \\ ${ }^{1}$ Departamento Física Teórica II, Facultad de Ciencias Físicas, Universidad Complutense de Madrid, 28040 Madrid, Spain \\ ${ }^{2}$ Departamento Física Atómica, Molecular y Nuclear, Facultad de Ciencias Físicas, Universidad Complutense de Madrid, \\ 28040 Madrid, Spain \\ (Received 20 July 2012; published 31 May 2013)
}

\begin{abstract}
Here we investigate the axial $w$ quasinormal modes of neutron stars for 18 realistic equations of state, most of them satisfying the $2 M_{\odot}$ condition. In particular, we study the influence of the presence of hyperons and quarks in the core of the neutron stars. We have obtained that $w$ modes can be used to distinguish between neutron stars with exotic matter and without exotic matter for compact enough stars. We present phenomenological relations for the frequency and damping times with the compactness of the neutron star for wI and wII modes showing the differences of the stars with exotic matter in the core. Also, we obtain a new phenomenological relation between the real part and the imaginary part of the frequency of the $w$ quasinormal modes, which can be used to estimate the central pressure of the neutron stars. Finally, we study the low compactness limit configuration of fundamental wII modes, and the influence of changes in the core-crust transition pressure. To obtain these results we have developed a new method based on the exterior complex scaling technique with variable angle.
\end{abstract}

DOI: 10.1103/PhysRevD.87.104042

PACS numbers: 04.40.Dg, 04.30.-w, 95.30.Sf, 97.60.Jd

\section{INTRODUCTION}

Considerable progress has been made in the last years on the development of gravitational wave detectors. Largescale interferometric gravitational wave detectors, such as LIGO, GEO, TAMA, and VIRGO, have reached the original design sensitivity, and are currently beginning to operate with frequencies between $1 \mathrm{~Hz}$ to $1 \mathrm{kHz}$. The sensitivity is continuously being enhanced [1]. First, detections are expected to happen within the next five years. These observations are of huge importance because they can be used to perform stringent tests of general relativity. But also because gravitational wave detection opens a new window to observations of the insights of numerous, and probably also new, astrophysical processes. Possible sources of gravitational waves are interacting black holes, coalescing compact binary systems, stellar collapses, and pulsars [2].

Neutron stars are major candidates to detectable gravitational wave sources because of their rich emission spectrum, which lays inside the frequency range of current detectors. A recent review about gravitational waves from neutron stars can be found in [3]. The study of the signature of the equation of state on gravitational radiation from neutron star has been developed in [4-9]. The first direct search for the gravitational-wave emission associated with oscillations of fundamental quadrupole mode excited by a pulsar glitch has been presented in [10].

Neutron stars are found inside pulsars, and are thought to originate after the collapse of massive star cores. The supernova explosion causes violent oscillations of the resulting compact star. Similarly, the coalescence of compact bodies like white dwarfs leaves behind an oscillating neutron star. The resulting excess of energy that causes the star to oscillate is radiated in the form of gravitational radiation [2].

It is well known that although the spectrum of neutron star oscillations is continuous, general perturbations cause the star to ring with concrete oscillation frequencies that dominates over the rest of possible frequencies for a certain period of time after the perturbation. These resonant frequencies can be studied introducing the concept of quasinormal modes, that is, eigenmodes of oscillation, which, although they do not form a complete set of functions in which to expand every possible perturbation evolution, are very useful in the determination of the eigenfrequencies for which the star tends to oscillate [11-13]. These eigenfrequencies are given by a complex number. The real part gives us the oscillation frequency of the mode, while the imaginary part gives us the inverse of the damping time. The quasinormal mode spectrum is quite dependent on the properties of the star, i.e., the equation of state.

Neutron stars are compact objects of very high density. Inside of them matter is found at extreme densities $\left(10^{15} \mathrm{~g} / \mathrm{cm}^{3}\right)$. Current theories predict a layer structure for the neutron star, essentially composed by the core and the crust. The properties of the crust are very different from those of the core, and it is thought that this region has a solid crystalline structure similar to a metal. Although the high density matter inside the star has exotic properties, the resulting relativistic fluid that composes the neutron star can be described as a perfect fluid. What we need to know is the equation of state for the neutron star matter; but beyond the nuclear densities this relation is not well understood. The inner part of the core is very model dependent, essentially because different populations of particle states may appear at those densities. The composition is not 
exactly known and several hypotheses exist $[14,15]$. Along the core of the star and especially in the core-crust interface, first order phase transitions are expected to be found in realistic equations of state. These phase transitions result in small discontinuities in the energy density of the star matter $[14,16]$.

The measurement of $1.97 M_{\odot}$ for PSR J1614-2230 imposes a stringent condition on the equations of state, in particular to those with exotic matter in the core [17]. Recently, several equations of state with exotic matter in the core satisfying the $2 M_{\odot}$ condition have been proposed [18-21].

In order to systematize the study of constraints placed by astrophysical observations on the nature of neutron star matter, several parametrizations of high-density equations of state (EOS) have been introduced: a piecewise polytropic approximation by Jocelyn et al. [22] and Lindblom spectral decomposition [23]. The advantage of these parametrizations is that they reduce the total amounts of parameters which modelize the equation of state to a more tractable quantity, allowing us to constrain with observational data the values of the parameters, and also usually they enhance a numerical integration scheme allowing better precision. Let us note that a very large family of equations of state can be fitted to these parametrizations with a very good precision. From a practical or numerical point of view, another possibility to describe the equation of state, satisfying local thermodynamic conditions, is a monotone piecewise cubic Hermite interpolation.

The detection of gravitational radiation from neutron stars can be a very useful tool to determine the neutron star equation of state. The extraction of the spectrum of quasinormal modes (frequency and damping time) from these signals could give important constrains to the equation of state, and, in consequence, information about the behavior of matter at densities beyond nuclear matter [24-30].

The theoretical study of the quasinormal mode spectrum considering different models of neutron stars with different equations of state is then well justified. The necessary formalism was developed first for quasinormal modes of black holes by Regge and Wheeler [31] and by Zerilli [32]. Quasinormal modes can be differentiated into polar and axial modes. In these papers it is found that the equation describing the quasinormal mode perturbation of the Schwarzschild metric is essentially a Schrödinger-like equation: the Regge-Wheeler equation for axial perturbations and the Zerilli equation for polar ones. For black holes, both types of modes are space-time modes. The formalism was studied in the context of neutron stars first by Thorne [33-37], Lindblom [38,39], and then reformulated by Chandrasekhar and Ferrari [40-42], and Kojima [43]. In neutron stars, axial modes are purely space-time modes of oscillation ( $w$ modes), while polar modes can be coupled to fluid oscillations (although a branch of $w$ modes can be found also in polar oscillations). In this paper we will consider only axial modes of oscillation.

The study of the quasinormal modes spectrum is complicated because of several reasons. Quasinormal modes can only be studied numerically. No analytical solutions are known for physically acceptable configurations of neutron stars. Another reason is that quasinormal modes are found as isolated points scattered on the complex plane, so that usually an exhaustive scan of this plane is necessary in order to find the complete spectrum. Also the numerical study is hindered by the very definition of quasinormal mode itself, that as we will see explicitly in the following sections, gives rise to diverging functions that oscillate an infinite number of times towards spatial infinity. These functions are not well handled numerically.

Several methods have been developed to deal with these and other difficulties. For a complete review on the methods see the review by Kokkotas and Schmidt [11].

Chandrasekhar and Ferrari [40-42] used a slowly damped approximation $(\operatorname{Im}[\omega] \ll \operatorname{Re}[\omega])$, which reduces the system of equations needed to resolve the problem, so that the damping time of the quasinormal mode can be given in terms of the real part. This method was also used in the determination of $w$ modes on polar perturbations of constant density neutron stars by Kojima et al. [44]. It is only valid for slowly damped modes, which are present in highly compact stars, and is not appropriate for realistic configurations, although it can be used to obtain a first approximation of the fundamental frequencies.

In an interesting paper [45], Kokkotas studied the axial spectrum integrating the exterior solution up to a finite radius, which allows us to impose the outgoing wave behavior to the exterior solution. The matching between the interior and exterior solutions results in the construction of a Wronskian at the matching surface, which must be zero when both solutions correspond to a quasinormal mode.

The Wentzel-Kramers-Brillouin method [46] can be used to approximate the outer solutions provided that no backscattering is found in the exterior region. This condition usually is satisfied when the imaginary part of the mode is small compared with the real part [47].

The phase function can be studied in order to deal with the oscillatory nature of the perturbation function. Studied for black holes by Chandrasekhar and Detweiller [48], the phase function plays a fundamental role in the numerical approach made by Andersson et al. in [47] and in [49] for neutron stars. The phase is usually a well-behaved function if there is not backscattering contamination in the numerical solution.

The divergence of the outgoing wave solution of the perturbation equations makes the avoidance of incoming wave contamination a difficult task. This problem was dealt with for black holes by Andersson in [50] by rotating the radial variable into a complex variable parallel to the antiStokes line. This approach was used for constant density 
neutron stars in $[47,49]$ together with the phase function approach. More recently, Samuelsson et al. [51] used a similar complex-radius approach for a constant density configuration which dealt with the divergence at infinity by integrating the Bondi-Sachs phase along a fixed path for the complexified radius parallel to the anti-Stokes line.

Another approach is based on the Leaver continued fraction method [52], which allows us to impose the outgoing wave behavior on the border of the star as a selfconsistent equation that can be satisfied iteratively [53]. This method has been used successfully with realistic models of neutron stars [24,26,30]. As commented above, for fluid modes there is also a branch of $w$ modes. As these are purely space-time modes, they do not couple to matter, and the inverse Cowling approximation can be used to obtain this part of the spectrum [49].

In this work we present a new approach to calculate quasinormal modes of realistic neutron stars. We make use of several well-known techniques, like the use of the phase for the exterior solution and the use of a complexified coordinate to deal with the divergence of the outgoing wave. We also introduce some new techniques not used before in this context: freedom in the angle of the exterior complex path of integration, implementation of realistic equations of state in a piecewise polytrope approximation or by monotone piecewise cubic Hermite interpolation, use of the COLSYS package to integrate all the system of equations at once with proper boundary and junction conditions and possibility of implementation of phase transition discontinuities. These new techniques allow us to enhance precision, to obtain more modes in shorter times, and also to study several realistic equations of state, comparing results for different compositions.

In Sec. II we will start presenting the well-known general formalism of quasinormal modes. In Sec. III we will present a study of the junction conditions for a general matching that can include first-order phase transitions or surface energy layers. In most of the results of the paper we will make use of the junction conditions without phase transitions or energy layers. In Sec. IV we describe the numerical method we have used to obtain our results, testing the algorithm to obtain already known modes for a simple model. In Secs. V, VI, and VII we present our results, focusing on 18 different equations of state: for pure nuclear matter (Sly, APR4), mixed hyperon-nuclear matter (GNH3, H1, H4, BGN1H1,WCS1, WCS2, BHZBM), hybrid stars (ALF2, ALF4,WSPHS3), hybrid stars with hyperons and quark color-superconductivity (BS1, BS2, BS3, BS4), and quark stars (WSPHS1, WSPHS2). In Sec. VIII we finish the paper with a summary of the main points of the work.

\section{QUASINORMAL MODE FORMALISM}

In order to fix the notation we will present a brief review of the formalism used to describe axial quasinormal modes. In the following we use geometrized units $(c=1, G=1)$.

We consider a static spherical space-time with metric $d s^{2}=e^{2 \nu} d t^{2}-e^{2 \lambda} d r^{2}-r^{2}\left(d \theta^{2}+\sin ^{2} \theta d \varphi^{2}\right)$. The matter inside of the star is considered perfect fluid with stress-energy tensor $T^{\mu \nu}=(p+\rho) u^{\mu} u^{\nu}-p g^{\mu \nu}$, where $p$ is the pressure, $\rho$ is the energy density and $u$ is the 4 -velocity. At zero order the equations obtained are well known. Inside the star we have

$$
\begin{gathered}
\frac{d m}{d r}=4 \pi r^{2} \rho, \\
\frac{d p}{d r}=-(\rho+p) \frac{m+4 \pi r^{3} p}{(r-2 m) r}, \\
\frac{d \nu}{d r}=-\frac{1}{\rho+p} \frac{d p}{d r},
\end{gathered}
$$

where we must provide an equation of state. Outside, we have the Schwarzschild solution with gravitational mass $M$.

Following the original papers [33-43], we make perturbations over this metric, expanding the perturbation in tensor harmonics, and taking into account only the axial perturbations. It can be demonstrated that the axial perturbations must satisfy the well-known Regge-Wheeler equation [32].

$$
\frac{d^{2} Z^{l k}}{d r_{*}^{2}}+\left[\omega^{2}-V(r)\right] Z^{l k}=0,
$$

where $l$ and $k$ are the spherical harmonic indexes, $r_{*}$ is the tortoise coordinate

$$
r_{*}=\int_{0}^{r} e^{\lambda-\nu} d r
$$

and the eigenfrequency of the axial mode is a complex number $\omega=\omega_{\operatorname{Re}}+i \omega_{\mathrm{Im}}$. The potential can be written as

$$
V(r)=\frac{e^{2 \nu}}{r^{3}}\left[l(l+1) r+4 \pi r^{3}(\rho+p)-6 m\right] .
$$

In this work we will consider only the $l=2$ case.

Axial oscillations do not modify the energy density or the pressure of the fluid. Hence, for axial modes, inside the star only the perturbations on the 4-velocity of the matter have to be taken into account (Lagrangian displacement).

Note that in general the perturbation function $Z$ is a complex function. Hence, we have a system of two real second-order differential equations (one for the real part $Z_{\mathrm{Re}}$, and another for the imaginary part $Z_{\mathrm{Im}}$ ).

$Z$ has to satisfy a set of boundary conditions that can be obtained from the following two requirements [40]: (i) the perturbation must be regular at the center of the star, and (ii) the resulting quasinormal mode must be a pure outgoing wave. 
The second requirement imposes the quasinormal modes to behave as purely outgoing waves at radial infinity. In general a quasinormal mode will be a composition of incoming and outgoing waves, i.e.,

$$
\lim _{r_{*} \rightarrow \infty} Z^{\text {in }} \sim e^{i \omega r_{*}}, \quad \lim _{r_{*} \rightarrow \infty} Z^{\text {out }} \sim e^{-i \omega r_{*}} .
$$

Note that, while the real part of $\omega$ determines the oscillation frequency of the wave, the imaginary part of the eigenvalue determines the asymptotic behavior of the quasinormal mode: If we call $\tau=1 / \omega_{I}$, the ingoing and outgoing modes will behave, respectively, as

$$
\lim _{r_{*} \rightarrow \infty} Z^{\text {in }} \sim e^{-r_{*} / \tau}, \quad \lim _{r_{*} \rightarrow \infty} Z^{\text {out }} \sim e^{r_{*} / \tau} .
$$

Outgoing quasinormal modes are divergent at radial infinity, while ingoing ones tend exponentially to zero as the radius grows.

In the next sections we will present our numerical method, which is able to deal with this problem.

\section{JUNCTION CONDITIONS}

To determine completely the problem, we must study the junction conditions between the interior solution and the exterior solution on the boundary of the star. The junction conditions between two space-times have been considered in classical works by Darmois [54], Lichnerowicz [55], O'Brien and Synge [56], and Israel [57], and, more recently, in [58-60]. In the context of relativistic rotating stars, it has been considered in [61-63].

We will impose the usual junction conditions. But, in order to have the possibility to study the influence of small changes in the core-crust transition pressure, we will include the more general case in which the star is surrounded by a surface layer of energy density.

We use Darmois conditions, the intrinsic formulation of these junction conditions, which imposes certain constrains on the continuity of the fundamental forms of the matching hypersurface $S$ [64].

This surface $S$ is defined by the points where the pressure is null, or more generally, constant. As we have seen previously the axial oscillations do not modify the pressure or the density, so the surfaces of constant pressure of the perturbed star are essentially the same as the surfaces of constant pressure of the static star.

Let us consider a surface $S$ of constant pressure inside the star, and a surface layer on top of $S$ described by a surface stress-energy tensor of a perfect fluid of the following form:

$$
T_{S}^{\mu \nu}(R)=\varepsilon u_{S}^{\mu}(R) u_{S}^{\nu}(R),
$$

where, $S$ is defined by the points where $r=$ const $=R, \varepsilon$ is the surface energy density of the fluid moving in $S$, and $u_{S}^{\mu}(R)$ is its velocity. The continuity of the first fundamental form gives us two conditions: the continuity of $\nu$

$$
\nu_{\text {int }}=\nu_{\text {ext }},
$$

and the continuity of perturbation function $Z$ :

$$
Z_{\text {int }}^{l k}=Z_{\mathrm{ext}}^{l k} \text {. }
$$

The second fundamental form conditions impose a jump in the pressure if $\varepsilon \neq 0$. Let the pressure in the inner part of the surface $S$, be $p_{\text {int }}$, and in the outer part be $p_{\text {ext }}$ with $p_{\text {ext }}<p_{\text {int }}$.

Making the same steps as with the first fundamental form, we obtain the following conditions for the zero order functions:

$$
\begin{gathered}
M_{\mathrm{ext}}=M_{\mathrm{int}}+4 \pi R^{2} \sqrt{1-\frac{2 M_{\mathrm{int}}}{R}} \varepsilon-8 \pi^{2} R^{3} \varepsilon^{2}, \\
\frac{M_{\mathrm{ext}}+4 \pi R^{3} p_{\mathrm{ext}}}{R^{2} \sqrt{1-\frac{2 M_{\mathrm{ext}}}{R}}}-\frac{M_{\mathrm{int}}+4 \pi R^{3} p_{\mathrm{int}}}{R^{2} \sqrt{1-\frac{2 M_{\mathrm{int}}}{R}}}=4 \pi \varepsilon .
\end{gathered}
$$

In this work we will consider $p_{\text {ext }}=0$. That is, the surface of star with pressure $p=p_{\text {int }}$ is being matched directly to the empty exterior surface of the star, in which case, $M_{\text {ext }}$ is just the Schwarzschild mass $M$. If we choose $p_{\text {int }}$ as the core-crust transition pressure, this matching condition is equivalent to approximating exterior layers of the star to a surface layer energy density that envelops the core of the star (surface crust approximation). The Eq. (12) can be interpreted as giving the total mass of the star to zero order $\left(M_{\text {ext }}\right)$, as the addition of three terms, the first one representing the core mass $\left(M_{\text {int }} \equiv 4 \pi \int_{0}^{R} \rho r^{2} d r\right)$, the second one representing the mass of the crust, and the third one being a negative bounding energy term. The radius of the resulting configuration will be the radius of the core of the star. Equation (13) can be seen as a condition for the determination of the radius of the core of the star when approximating the crust of the star to a thin surface energy density.

In the surface crust approximation, these relations allow us to use the core-crust transition pressure $p_{\text {int }}$, as a new parameter of the resulting configuration.

Up to first order in the perturbative expansion we obtain junction conditions for the axial functions. Essentially we obtain the following condition for the derivative of the $Z$ function:

$$
\left.\frac{d Z^{l k}}{d r}\right|_{\mathrm{ext}}=\left.\frac{e^{\lambda_{\mathrm{ext}}}}{e^{\lambda_{\mathrm{int}}}} \frac{d Z^{l k}}{d r}\right|_{\mathrm{int}}+\left[\frac{e^{\lambda_{\mathrm{ext}}}}{e^{\lambda_{\mathrm{int}}}}-1\right] \frac{Z^{l k}}{R} .
$$

Together with condition (11) we have enough information to perform correctly the matching of the perturbation.

Let us note that, the usual treatment without surface crust approximation is recovered by making in the previous conditions $p_{\text {int }}=p_{\text {ext }}=0$ and $\varepsilon=0$, in which case the continuity of the metric functions and of their derivatives is obtained. This is what we do in almost the entire paper except in the final part of Sec. VII, where the influence of the changes in the core-crust transition pressure is analyzed. 


\section{NUMERICAL METHOD}

In this section we will explain the numerical method we use to obtain outgoing quasinormal modes of realistic stars. We implement this method into different Fortran programs and routines, making use of the COLSYS package [65] to solve numerically the differential equations. The advantage of this package is that it allows the utilization of quite flexible multiboundary conditions, that, as we will see in the following sections, are important in our method of quasinormal mode determination. On the other hand, COLSYS allows us to construct an adaptative mesh of points depending of the precision we would like to achieve. In this manner, the more precision we need on the perturbation function, the more points COLSYS introduces into the mesh. The static solution is calculated at the same time as the perturbation, so the static solution functions will be also adapted to the precision we require for the Regge-Wheeler function. COLSYS additionally allows the use of the continuation method: we can use as an initial guess for a new numerical solution another numerical solution with parameters close enough to the parameters of the new solution.

We will start explaining how the outgoing modes are determined in the exterior region of the metric.

Quasinormal modes present an oscillating part given by the real part of the eigenfrequency, that remains towards infinity, as can be seen in relation (7).

In order to deal with this issue we will follow [50,51], and we will study the phase function. The phase function (logarithmic derivative of the $Z$ function),

$$
g=\frac{Z^{\prime}}{Z}
$$

does not oscillate towards asymptotic infinity.

The other important issue we have seen is that quasinormal modes diverge towards spatial infinity because of their pure outgoing wave behavior. This problem can be treated using the phase function together with a rotation of the radial coordinate into the complex plane.

This technique of complexification of the integration variable is called exterior complex scaling [66-68]. An analytical extension of the $g$ function, which is a function of $r$, is made into a $g$ function that is dependent of the generalized complex coordinate. This new function has no physical meaning, but the eigen-values obtained by integrating the equation along the extended radius will remain unaltered.

We parametrize the complex coordinate along a line in the complex plane. We will take a straight line with an angle $\alpha$ with respect to the real axis.

$$
r=R+y e^{-i \alpha},
$$

with $y \in[0, \infty)$. The $g$ function has the same limits in the case of a purely outgoing or a purely ingoing wave than with the real coordinate. But the mixed waves now depend on the angle of the path of integration $\alpha$.

In our method, the angle of the integration path is treated as a free parameter of the solution. This angle can be chosen appropriately to increase precision and integration time, but always remaining in the region where $g \rightarrow-i \omega$. This boundary condition imposes to the obtained solution a

TABLE I. Frequency and damping time of the trapped modes $(t)$, the spatial modes and the interface modes (i), in central density units, for axial $l=2$ modes, $2 M / R=0.885$.

\begin{tabular}{lccc}
\hline \hline$\omega_{\operatorname{Re}}$ & $\omega_{\operatorname{Im}}$ & $\omega_{\operatorname{Re}}$ & $\omega_{\operatorname{Im}}$ \\
\hline$(t) 0.21386387$ & $2.4320459 \times 10^{-9}$ & 1.68347876 & $6.0243716 \times 10^{-2}$ \\
$(t) 0.29101153$ & $7.7472523 \times 10^{-8}$ & 1.76704836 & $6.1930099 \times 10^{-2}$ \\
$(t) 0.36799864$ & $1.0725279 \times 10^{-6}$ & 1.85066583 & $6.3390024 \times 10^{-2}$ \\
$(t) 0.44463493$ & $9.5187522 \times 10^{-6}$ & 1.93429817 & $6.4699961 \times 10^{-2}$ \\
$(t) 0.52063903$ & $6.3393194 \times 10^{-5}$ & 2.01793447 & $6.5897686 \times 10^{-2}$ \\
0.59557900 & $3.3806006 \times 10^{-4}$ & 2.10157798 & $6.7019361 \times 10^{-2}$ \\
0.66896067 & $1.4322666 \times 10^{-3}$ & 2.18522398 & $6.8080510 \times 10^{-2}$ \\
0.74090953 & $4.5080077 \times 10^{-3}$ & 2.26888071 & $6.9098878 \times 10^{-2}$ \\
0.81280272 & $1.0135742 \times 10^{-2}$ & 2.35254774 & $7.0076042 \times 10^{-2}$ \\
0.88590397 & $1.7259264 \times 10^{-2}$ & 2.43622599 & $7.1018102 \times 10^{-2}$ \\
0.96037307 & $2.4480195 \times 10^{-2}$ & (i) 1.45115909 & $5.4011419 \times 10^{-2}$ \\
1.03608110 & $3.1027484 \times 10^{-2}$ & (i) 1.73433905 & $1.3945066 \times 10^{-2}$ \\
1.11308152 & $3.6685221 \times 10^{-2}$ & (i) 2.01106806 & $2.2902687 \times 10^{-2}$ \\
1.19147020 & $4.1559313 \times 10^{-2}$ & (i) 2.29393679 & $3.1975625 \times 10^{-2}$ \\
1.27122330 & $4.5838031 \times 10^{-2}$ & (i) 2.57982976 & $4.1067802 \times 10^{-2}$ \\
1.35219268 & $4.9636532 \times 10^{-2}$ & (i) 2.86633683 & $5.0155809 \times 10^{-2}$ \\
1.43415622 & $5.2976144 \times 10^{-2}$ & (i) 3.15274466 & $5.9234204 \times 10^{-2}$ \\
1.51685560 & $5.5841641 \times 10^{-2}$ & (i) 3.43712011 & $6.8307813 \times 10^{-2}$ \\
1.60003382 & $5.8244121 \times 10^{-2}$ & & \\
\hline \hline
\end{tabular}




\section{J. L. BLÁZQUEZ-SALCEDO et al.}

purely outgoing wave behavior. Exterior complex scaling with variable angle is widely used in other contexts like atomic and molecular physics, where it gives excellent results [69].

The use of the phase and exterior complex scaling with variable angle allows us to compactify, so we can impose the outgoing quasinormal mode behavior as a boundary condition at infinity without using any cutoff for the radial coordinate.

Inside the star the Regge-Wheeler equation (4) can be solved without any problem. The perturbation function is oscillating, but the number of oscillations will be finite between $r=0$ and $r=R$.

We must provide an equation of state. We implement the realistic EOS in two different ways: (i) A piecewise polytrope approximation, done by Read et al. [22]. In this approximation the equation of state is approximated by a polytrope in different density-pressure intervals. For densities below the nuclear density, the SLy equation of state is considered. (ii) A piecewise monotone cubic Hermite interpolation satisfying the local thermodynamic condition [70].

We generate two independent solutions of the ReggeWheeler equation inside the star. The junction conditions (11) and (14) can be written in the form of a determinant making use of these two solutions together with the exterior phase function. The determinant can be calculated numerically from the integrated solutions.

We must explore the plane $\left(\omega_{\mathrm{Re}}, \omega_{\mathrm{Im}}\right)$ looking for null determinants. This process can be automated so that the program looks for minima of the determinant on the plane.

We have made several tests on our method using simpler equations of state. We successfully reproduce data from previous works. For example, in Table I we show our results for a star of constant density and $2 M / R=0.885$, in perfect agreement with [51].

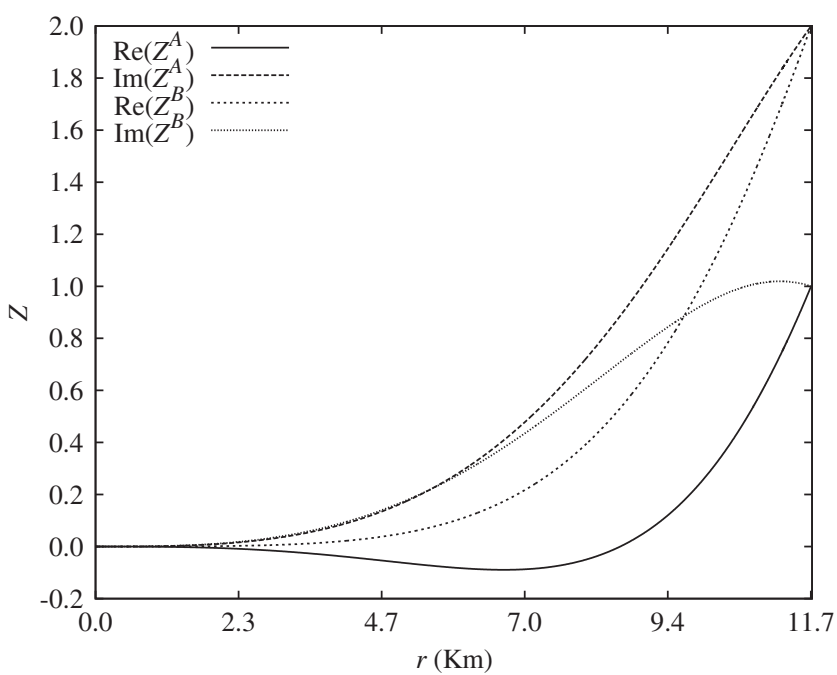

FIG. 1. Example of the Regge-Wheeler function inside the star vs radius.
PHYSICAL REVIEW D 87, 104042 (2013)

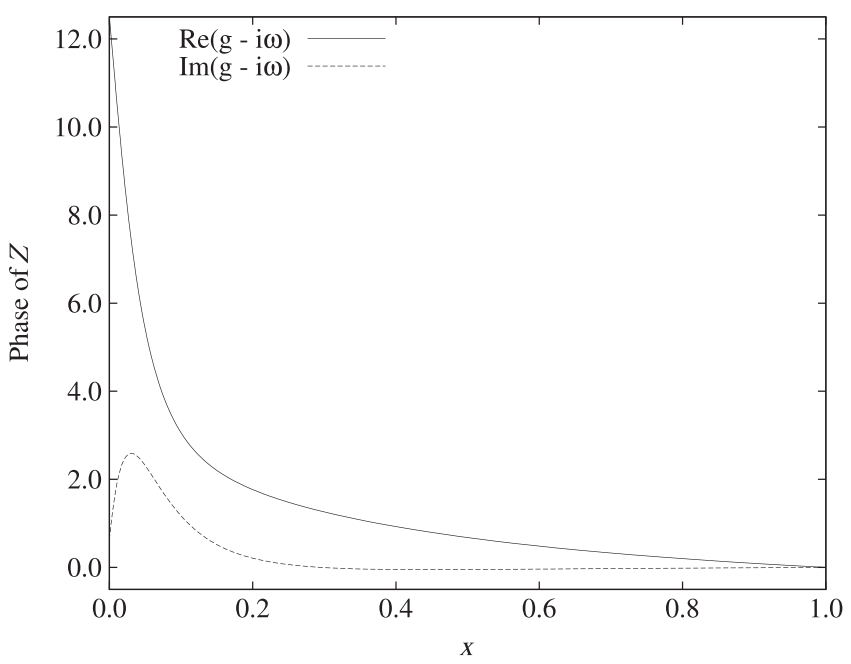

FIG. 2. Example of phase function $g$ outside the star vs the compactified rotated coordinate $x$.

In Figs. 1 and 2 we show an example of calculated perturbation functions. These functions correspond to a star of $1.4 M_{\odot}$, with Sly equation of state. The quasinormal mode is the first axial mode $w_{I}$ with frequency $\nu=8.034 \mathrm{kHz}$ and damping time $\tau=29.31 \mu \mathrm{s}$. In Fig. 1 we plot the real and imaginary parts of the two interior solutions for $Z$ vs $r$. In Fig. 2 we plot the real and imaginary parts of the calculated phase function outside the star vs the compactified rotated coordinate $x$.

In the next sections we will show the results obtained for realistic equations of state.

\section{RESULTS FOR REALISTIC EQUATIONS OF STATE}

In this section we present our results for the axial quasinormal modes of neutron stars with realistic equations of state. We consider a wide range of equations of state in order to study the influence of exotic matter in the core of the star on the axial quasinormal modes.

Using the parametrization presented by Read et al. [22], which is implemented in our code, we can study the 34 equations of state they considered. For this paper we have used, following their notation, SLy, APR4, BGN1H1, GNH3, H1, H4, ALF2, ALF4.

After the recent measurement of the $1.97 M_{\odot}$ for the pulsar PSR J164-2230, several equations of state have been proposed. These new equations allow the presence of exotic matter in the core of the neutron star, and the maximum mass for stable configurations is over this value. We have considered the following ones: two equations of state presented by Weissenborn et al. with hyperons in [20]; we call them WCS1 y WCS2, three EOS presented by Weissenborn et al. with quark matter in [21]; we call them WSPHS1, WSPHS2, WSPHS3, four equations of state presented by Bonanno and Sedrakian in [19]; we 
call them BS1, BS2, BS3, BS4, and one EOS presented by Bednarek et al. in [18]; we call it BHZBM.

In Fig. 3 we plot the 18 equations of state we have studied in order to give an idea of the range considered. Let us describe briefly the characteristics of the equations of state we have used: For plain $n p e \mu$ nuclear matter, we use

(i) SLy [71] with npe $\mu$ using a potential method to obtain the EOS.

(ii) APR4 [72] with $n p e \mu$, obtained using a variational method.

For mixed hyperon-nuclear matter we use

(i) GNH3 [73], a relativistic mean-field theory EOS containing hyperons.

(ii) $\mathrm{H} 1$ and $\mathrm{H} 4$ [74], two variants of the $\mathrm{GNH} 3$ equation of state.

(iii) BGN1H1 [75], an effective-potential equation of state including hyperons.

(iv) WCS1 and WCS2 [20], two equations of state with hyperon matter, using "model $\sigma \omega \rho \phi$ ", and considering ideal mixing, $\mathrm{SU}(6)$ quark model, and the symmetric-antisymmetric couplings ratio $\alpha_{v}=1$ and $\alpha_{v}=0.2$, respectively.

(v) BHZBM [18], a nonlinear relativistic mean field model involving baryon octet coupled to meson fields.

For hybrid stars we use

(i) ALF2 and ALF4 [76], two hybrid EOS with mixed APR nuclear matter and color-flavor-locked quark matter.

(ii) WSPHS3 [21], a hybrid star calculated using the bag model, mixed with NL3 RMF hadronic EOS. The parameters employed are $B_{\text {eff }}^{1 / 4}=140 \mathrm{MeV}$, $a_{4}=0.5$, and a Gibbs phase transition.

For Hybrid stars with hyperons and quark color superconductivity we use

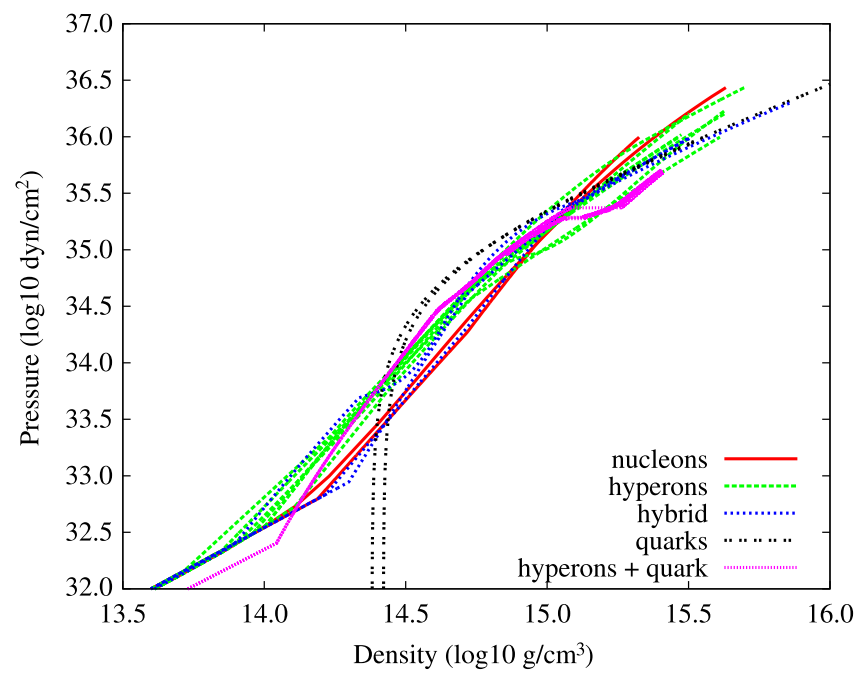

FIG. 3 (color online). Pressure vs density in logarithmic scale for the 18 equations of state considered, in the high density region. (i) BS1, BS2, BS3, and BS4, [19], four equations of state calculated using a combination of phenomenological relativistic hypernuclear density functional and an effective NJL model of quantum chromodynamics. The parameters considered are vector coupling $G_{V} / G_{S}=0.6$ and quark-hadron transition density $\rho_{\text {tr }} / \rho_{0}$ equal to $2,3,3.5$, and 4 , respectively, where $\rho_{0}$ is the density of nuclear saturation.

For quark stars we use

(i) WSPHS1 and WSPHS2 [21]. The first equation of state is for unpaired quark matter, and we have considered the parameters $B_{\text {eff }}^{1 / 4}=123.7 \mathrm{MeV}$, $a_{4}=0.53$. The second equation of state considers quark matter in the CFL phase (paired). The parameters considered are $B_{\mathrm{eff}}^{1 / 4}=130.5 \mathrm{MeV}, a_{4}=0.66$, $\Delta=50 \mathrm{MeV}$.

\section{A. Nuclear and hyperon matter}

We will start studying the impact of hyperon matter on the quasinormal mode spectrum, comparing with configurations with plain nuclear matter EOS.

In Figs. 4 and 5 we plot the mass-central pressure and mass-radius relation, respectively. For the study of the quasinormal modes we have considered only stable configurations below the maximum mass of each equation of state. It can be seen that the introduction of a hyperon core changes the mass radius relation and we will see that this has an imprint on the quasinormal mode spectrum of these configurations. Note that all the EOS considered are near or beyond the $1.97 M_{\odot}$ limit, except the BGN1H1 and $\mathrm{H} 1$ equations of state, which are included for comparison. In the following we will consider only stable configurations

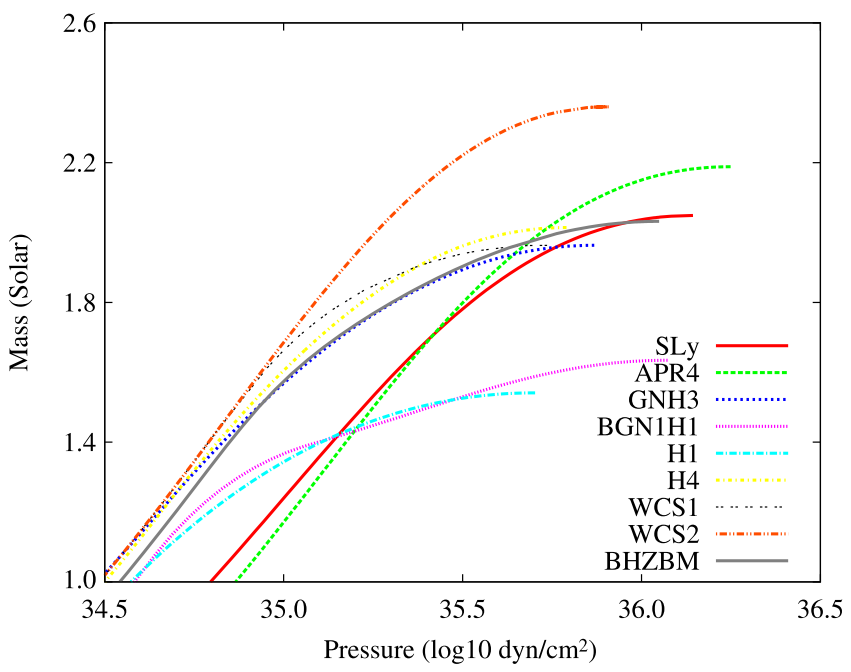

FIG. 4 (color online). Mass vs central pressure for the equations of state considered in Sec. VA. We plot configurations up to the maximum stable mass. Note that all the EOS have a maximum mass nearby or beyond $2 M_{\odot}$, except $\mathrm{H} 1$ and BGN1H1, which are included for comparison. 


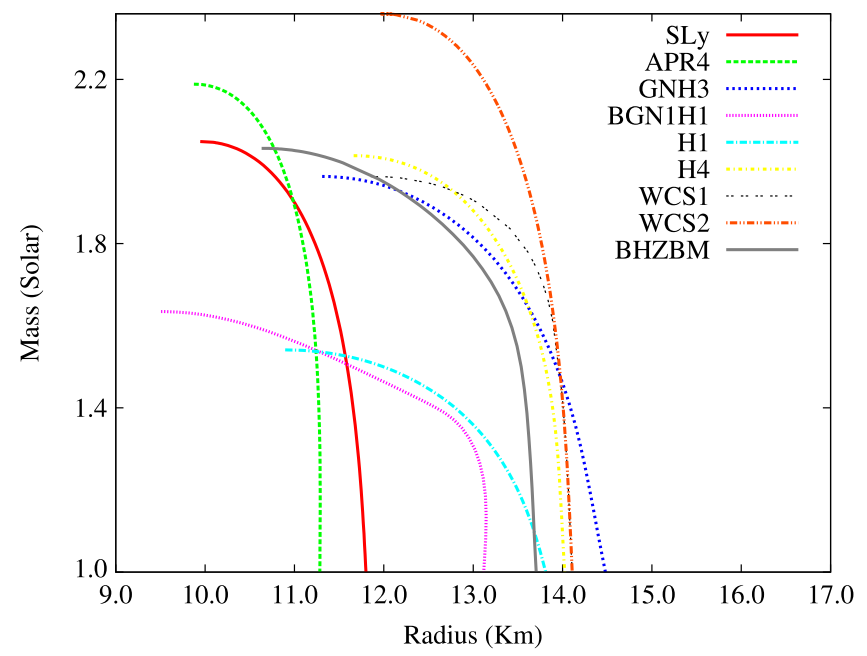

FIG. 5 (color online). Mass vs radius for the equations of state considered in Sec. VA. Typically, stars with hyperon matter have bigger radius than nuclear matter stars of the same mass.

between $1 M_{\odot}$ and the maximum mass of the particular equation of state. We will study the fundamental and first excited wI modes, as well as the fundamental wII mode.

Fundamental wI mode: In Figs. 6 and 7, we plot the frequency and damping time of the fundamental wI mode as a function of the central pressure of the star in logarithmic scale, for the different equations of state. There is no significant difference between the two pure nuclear matter equations of state considered, except for the bigger maximum mass of the APR4 EOS. On the other hand, we observe that the presence of hyperon condensates has a clear impact on the frequencies of the fundamental

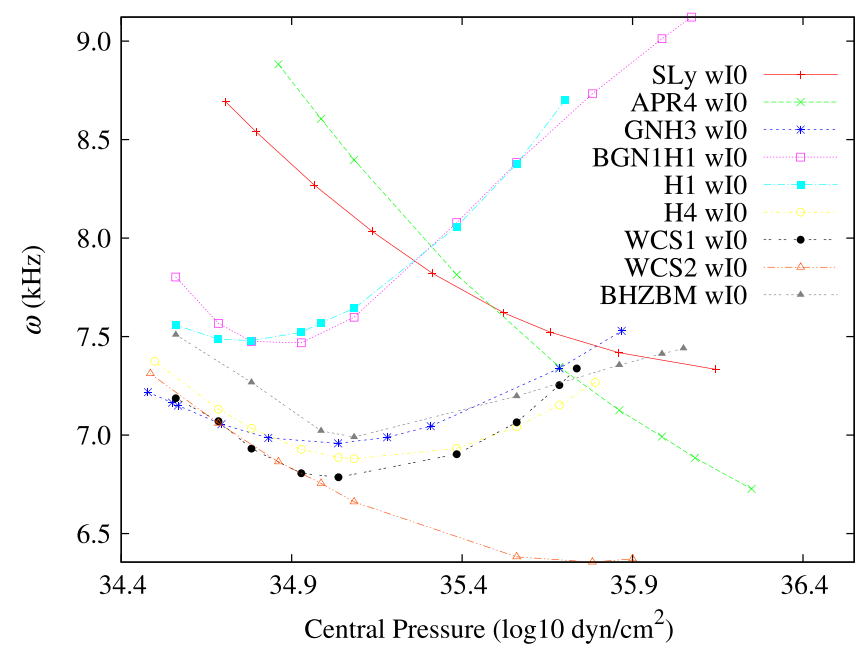

FIG. 6 (color online). Frequency of the fundamental wI mode vs central pressure in logarithmic scale, for the EOS considered in Sec. VA. While there is no significant difference between the SLy and the APR4, the hyperon core changes the relation of the frequency with the central pressure for high densities ( $p_{c}$ above $\sim 10^{35} \mathrm{dyn} / \mathrm{cm}^{2}$ ).
PHYSICAL REVIEW D 87, 104042 (2013)

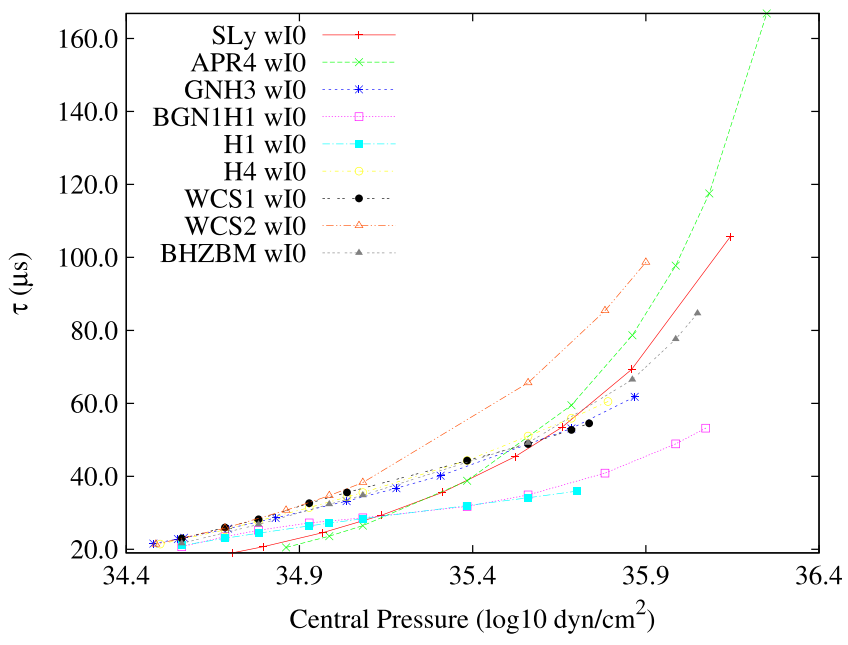

FIG. 7 (color online). Damping time of the fundamental wI mode vs central pressure in logarithmic scale, for the EOS considered in Sec. VA. The behavior is quite similar for all the equations of state considered. At high density, the lowest values of the damping time are reached by the H1 and BGN1H1 EOS.

$w$ modes. Note that the hyperon core is changing the frequency relation for high mass configurations, increasing with the central pressure above $\sim 10^{35} \mathrm{dyn} / \mathrm{cm}^{2}$, where the hyperon phases are more important due to the high density of the star core. Essentially, this effect is related to the softening of the EOS by the introduction of hyperon matter at the inner regions of the star.

This result is in agreement with a recent work by Chatterjee and Bandyopadhyay [29].

In order to use future observations of gravitational waves to estimate the mass and the radius of the neutron star, as well as to discriminate between different families of equations of state, we obtain empirical relations between the frequency and damping time of quasinormal modes and the compactness of the star, following $[4,25,77,78]$. In Fig. 8 we present the frequency of the fundamental mode scaled to the radius of each configuration. It is interesting to note that even with this scaling, the softest equations of state that include hyperon matter, $\mathrm{H} 1$ and $\mathrm{BGN} 1 \mathrm{H} 1$, present a quite different behavior than the rest of EOS considered. Nevertheless, as the detection of the recent $2 M_{\odot}$ pulsar suggest, these two particular EOS cannot be realized in nature, so the behavior of the scaled frequency is quite similar for hyperon matter or plain nuclear matter stars.

A linear fit can be made to each equation of state. We fit to the following phenomenological relation:

$$
\omega(K h z)=\frac{1}{R(K m)}\left(A \frac{M}{R}+B\right)
$$

In Table II we present the fit parameters $A, B$ for each one of the equations of state studied. For the Sly and for the APR4 equations very similar results are obtained. 


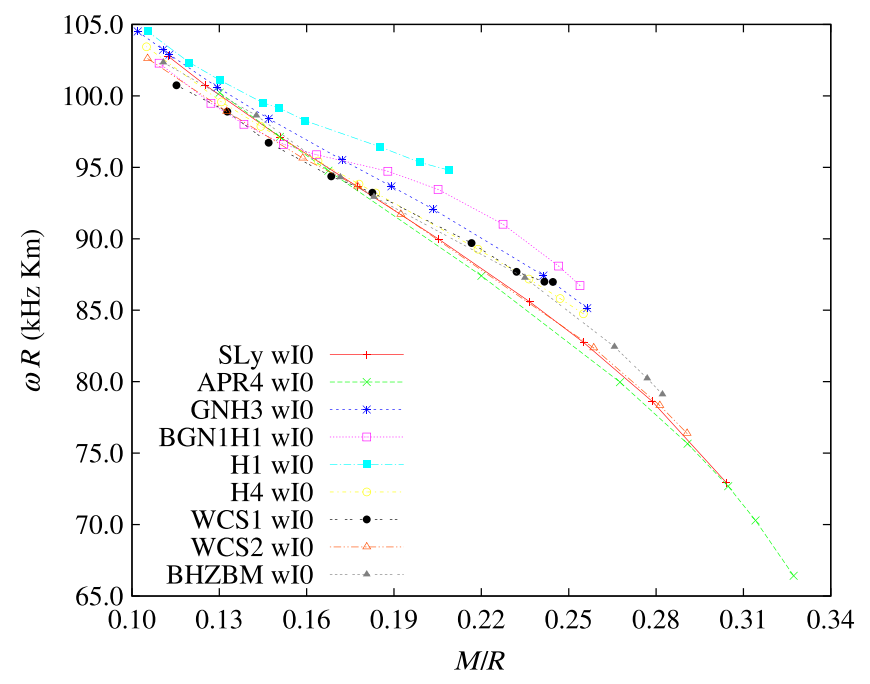

FIG. 8 (color online). Scaled frequency of the fundamental wI mode vs $M / R$, for the EOS considered in Sec. VA. Except for $\mathrm{H} 1$ and BGN1H1 EOS, which present a quite different behavior, all the scaled frequencies can be fitted to a linear relation with the compactness (17). The fits can be found in Table II.

The empirical parameters for SLy and APR4 are compatible with the empirical relation obtained by Benhar et al. [78] for six equations of state.

In general, for hyperon matter EOS, we still obtain a linear relation, but with slightly different parameters, as can be seen in Table II. As commented before, quite remarkable is the case of the $\mathrm{H} 1$ and $\mathrm{BGN} 1 \mathrm{H} 1$, where the linear relation is almost lost.

Our results show that the introduction of hyperon matter changes very little the dependence of the frequency with the compactness. Even for $M / R$ bigger than 0.18 , the difference between equations of state with and without hyperon matter is quite small. As these empirical relations depend minimally on the equation of state, they could be used, applying the technique from [77] to measure the radius of the neutron star and constrain the equation of state.

In Fig. 9 we present the damping time of the fundamental mode scaled to the mass of each configuration. In this case, the results can be fitted to an empirical quadratic relation on $M / R$, as follows

$$
\frac{10^{3}}{\tau(\mu s)}=\frac{1}{M\left(M_{\odot}\right)}\left[a\left(\frac{M}{R}\right)^{2}+b \frac{M}{R}+c\right] \text {. }
$$

In Table II we present the fit parameters $a, b$, and $c$. Note that for SLy and APR4 the fits are quite similar, and in accordance with the results obtained in [78]. All of the equations of state are well fitted to a quadratic relation with the compactness. For compactness beyond 0.18 the presence of hyperon matter slightly changes the dependence of the scaled damping time with $M / R$, specially for the BGN1H1 EOS.

First excited wI mode.-In Figs. 10 and 11 we plot the scaled frequency and damping time for the first excited wI modes. We make fits to the empirical relations (17) and (18). In Table III we present the corresponding empirical parameters.

Fundamental wII mode.-In Fig. 12 we plot the frequency of the fundamental wII mode scaled with the mass vs the metric function $e^{2 \lambda(R)}$. It can be seen that for all the equations of state studied, there is a minimal compactness below which the wII mode does not exist. The scaled frequency is quite linear with $e^{2 \lambda(R)}$ nearby this minimal compactness. Our numerical scheme allows us to obtain explicitly these limit configurations with vanishing real part of the mode with a precision of the order of $10^{-5}$. The limit configuration has compactness between $M / R=$ 0.1044 and $M / R=0.1066$, so it is quite independent of the particular equation of state, and in consequence independent of the presence of hyperon matter. For example, the GNH3 equation of state limit configuration has compactness $M / R=0.1051$, and for the BGN1H1, $M / R=$ 0.1061 , and the SLy has compactness $M / R=0.1056$ and the APR4 compactness $M / R=0.1046$. The reason why the limit configuration is independent of the EOS is because, for these values of the compactness, the mass of the stars considered are below $1 M_{\odot}$. In these low mass configurations the presence of hyperons at the core is small or null, depending of the equation of state, and so we are comparing plain nuclear matter stars with slightly different EOS at the core.

These limit configurations were conjectured by extrapolation in Wen et al. in [30]. Our results are in agreement with their extrapolation.

TABLE II. Fits for the wI0 modes. Parameters $A$ and $B$ correspond to the linear empirical relation for the frequency (17). Parameters $a, b$, and $c$ correspond to the quadratic empirical relation for the damping time (18).

\begin{tabular}{|c|c|c|c|c|c|c|c|c|c|}
\hline wI0 & SLy & APR4 & GNH3 & BGN1H1 & H1 & $\mathrm{H} 4$ & WCS2 & WCS1 & BHZBM \\
\hline 11 & $-148.7 \pm 4.5$ & $-164.2 \pm 5.8$ & $-122.1 \pm 1.4$ & $95.5 \pm 5.4$ & $-89.9 \pm 5.1$ & $120.6 \pm 1.8$ & $139.7 \pm 3.6$ & $-107.1 \pm 2.2$ & $-132.8 \pm 3.6$ \\
\hline$B$ & $119.8 \pm 1.0$ & $122.4 \pm 1.4$ & $116.67 \pm 0.25$ & $111.9 \pm 1.0$ & $113.06 \pm 0.81$ & $115.50 \pm 0.35$ & $117.83 \pm 0.75$ & $112.81 \pm 0.43$ & $117.35 \pm 0.78$ \\
\hline$x^{2}$ & 0.760 & 0.039 & 1.56 & 0.689 & 0.260 & 0.0812 & 0.430 & 0.096 & 0.388 \\
\hline$\hat{a}$ & $-1221 \pm 22$ & $-1112 \pm 19$ & $-1444 \pm 23$ & $-1690 \pm 49$ & $-1539 \pm 42$ & $-1385 \pm 47$ & $-1244 \pm 23$ & $-1450 \pm 64$ & $-1401 \pm 41$ \\
\hline$b$ & $365.1 \pm 9.3$ & $331.1 \pm 9.0$ & $425.4 \pm 8.3$ & $506 \pm 18$ & $448 \pm 13$ & $414 \pm 17$ & $371.4 \pm 9.6$ & $437 \pm 24$ & $418 \pm 17$ \\
\hline$c$ & $21.63 \pm 0.89$ & $24.16 \pm 0.94$ & $17.87 \pm 0.68$ & $11.2 \pm 1.6$ & $16.6 \pm 1.0$ & $18.2 \pm 1.5$ & $21.4 \pm 0.9$ & $16.2 \pm 2.1$ & $17.9 \pm 1.6$ \\
\hline$\chi^{2}$ & 0.050 & 0.034 & 0.027 & 0.084 & 0.016 & 0.091 & 0.044 & 0.083 & 0.097 \\
\hline
\end{tabular}




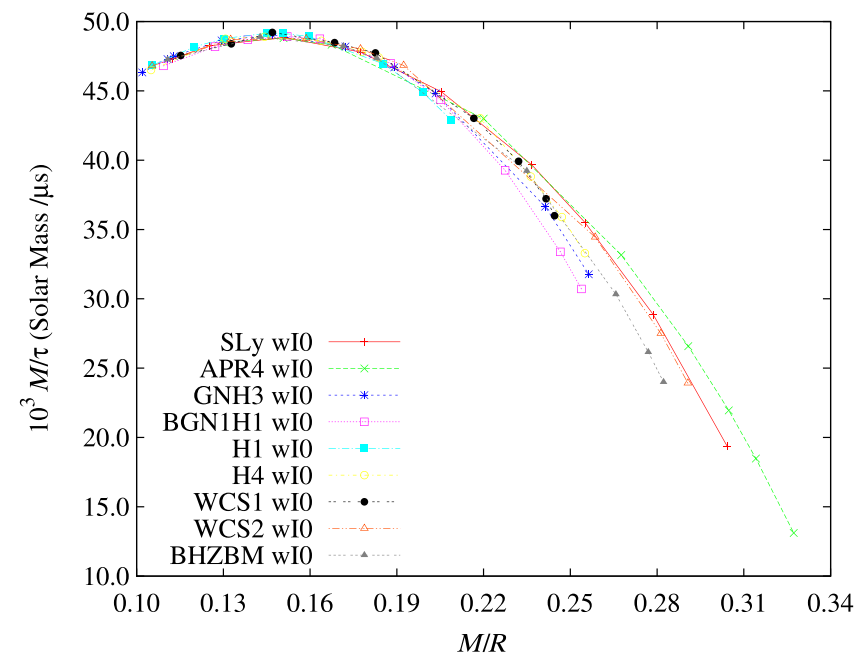

FIG. 9 (color online). Scaled damping time of the fundamental wI mode vs $M / R$, for the EOS considered in Sec. VA. The scaled damping times can be fitted to a quadratic relation with the compactness (18). The fits can be found in Table II.

In Fig. 13 we show the damping time of the wII mode scaled with the mass. Although for low compactness the damping time is quite independent of the equation of state and linear in $M / R$ for the reasons mentioned before, for compactness above 0.18 it is very sensitive to the presence of hyperon matter, changing completely the linear behavior. The damping time does not vanish in the limit compactness as the frequency does. On the contrary, it tends to a limit $\tau$.

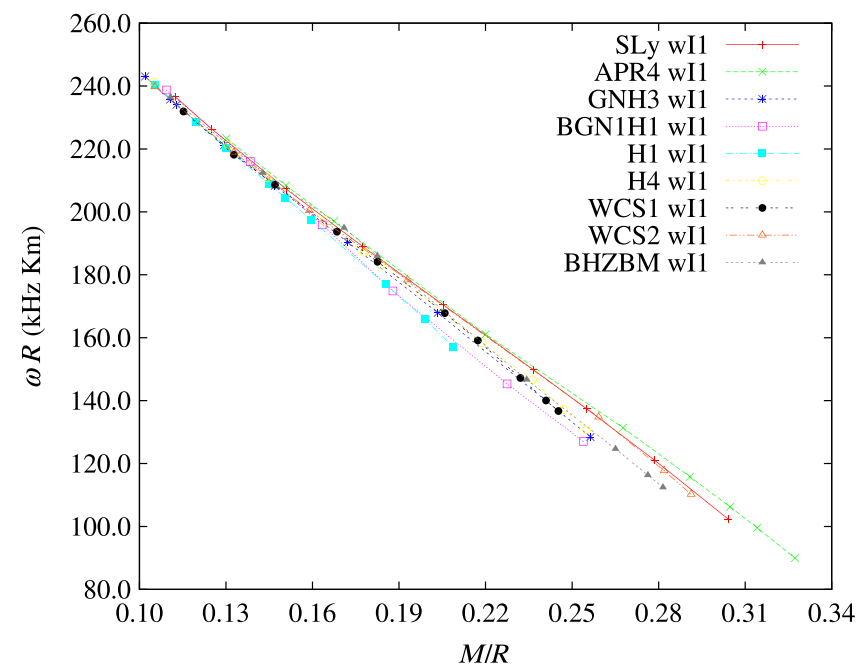

FIG. 10 (color online). Scaled frequency of the first excited wI mode vs $M / R$, for the EOS considered in Sec. VA. In this case the linear relation (17) is found for all these EOS. If we exclude the $\mathrm{H} 1$ and $\mathrm{BN} 1 \mathrm{H} 1 \mathrm{EOS}$, the presence of hyperons is not affecting strongly the scaled frequency. The fits can be found in Table III.
PHYSICAL REVIEW D 87, 104042 (2013)

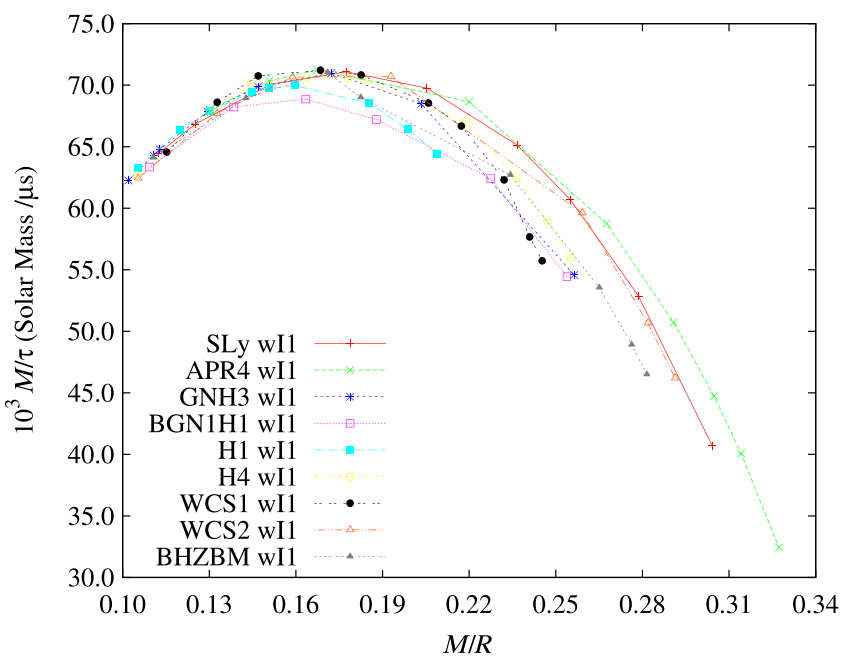

FIG. 11 (color online). Scaled damping time of the first excited wI mode vs $M / R$, for the EOS considered in Sec. VA. The quadratic relation (18) is valid for every EOS considered. The empirical parameters are more sensitive to the hyperon core, especially at high densities. The fits can be found in Table III.

\section{B. Quark matter}

In this subsection we will present our results for the axial quasinormal modes of compact stars with quark matter at the core. We consider the following equations of state: ALF2, ALF4, WSPHS1-3, BS1-4, already described previously. In the following figures we will always include the results for the SLy EOS for comparison.

In Fig. 14 we present the equations of state at the high density region. In Fig. 15 we present the mass-radius relation for these equations of state and in Fig. 16 the mass-central pressure relation. Only stable configurations below the maximum mass of each equation of state have been considered. Note that all the equations of state considered are near or above the $1.97 M_{\odot}$ limit. We will repeat the analysis for these new equations, studying the fundamental and first excited wI modes, and, finally, the fundamental wII mode.

Fundamental wI mode.-In Fig. 17 we plot the frequency of the fundamental wI mode as a function of the central pressure of the star in logarithmic scale. In Fig. 18 we make a similar plot for the damping time of these configurations. Note the similitude between the SLy modes and the ALF4 modes for both the frequency and damping time. These two equations of state cannot be differentiated by the observation of the fundamental $w$ mode. This is not the case if the EOS for hybrid matter is softer, as it happens for the other EOS considered, which present smaller frequencies.

In Figs. 17 and 18 we also plot the fundamental mode for the two pure quark matter EOS considered (WSPHS1 and WSPHS2). The configurations near the maximum mass limits of these EOS have much lower frequencies than the rest of the EOS studied, around $5.5 \mathrm{kHz}$. 
TABLE III. Fits for the wI1 modes. Parameters $A$ and $B$ correspond to the linear empirical relation for the frequency (17). Parameters $a, b$, and $c$ correspond to the quadratic empirical relation for the damping time (18).

\begin{tabular}{|c|c|c|c|c|c|c|c|c|c|}
\hline wI1 & SLy & APR4 & GNH3 & BGN1H1 & $\mathrm{H} 1$ & $\mathrm{H} 4$ & WCS2 & WCS1 & BHZBM \\
\hline$A$ & $-689.5 \pm 5.2$ & $-667.9 \pm 3.9$ & $-736.5 \pm 6.8$ & $-779.4 \pm 6.8$ & $-797 \pm 12$ & $-703.7 \pm 5.5$ & $-684.2 \pm 6.8$ & $-723.5 \pm 7.0$ & $-727.7 \pm 6.2$ \\
\hline$B$ & $312.5 \pm 1.1$ & $309.35 \pm 0.97$ & $317.2 \pm 1.4$ & $323.3 \pm 1.4$ & $324.4 \pm 0.7$ & $316.2 \pm 1.1$ & $310.5 \pm 1.5$ & $315.3 \pm 1.4$ & $317.5 \pm 1.3$ \\
\hline$\chi^{2}$ & 0.997 & 0.670 & 0.372 & 1.986 & 0.18 & 0.731 & 1.564 & 0.955 & 1.144 \\
\hline a & $-1843 \pm 55$ & $-1792 \pm 65$ & $-2062 \pm 25$ & $-1771 \pm 123$ & $-2274 \pm 20$ & $-2020 \pm 71$ & $-1863 \pm 78$ & $-2635 \pm 150$ & $-1854 \pm 79$ \\
\hline$b$ & $650 \pm 23$ & $646 \pm 30$ & $690.9 \pm 8.9$ & $582 \pm 45$ & $725.8 \pm 6.6$ & $687 \pm 26$ & $656 \pm 32$ & $879 \pm 55$ & $627 \pm 32$ \\
\hline$c$ & $14.3 \pm 2.2$ & $13.7 \pm 3.2$ & $13.10 \pm 0.73$ & $21.1 \pm 3.9$ & $12.02 \pm 0.51$ & $12.7 \pm 2.3$ & $14 \pm 29$ & $3.1 \pm 4.8$ & $17.4 \pm 3.0$ \\
\hline$\chi^{2}$ & 0.311 & 0.510 & 0.380 & 0.393 & 0.00374 & 0.209 & 0.401 & 0.520 & 0.361 \\
\hline
\end{tabular}

In these figures we observe that the frequency and damping times have a clear dependence on the EOS when represented against the central pressure. All the EOS with quark matter at the core present a characteristic behavior, with frequencies below those of the plain nuclear matter configurations, except the ALF4 EOS, which mimics the SLy EOS almost entirely.

In Fig. 19 we present the frequency of the fundamental mode scaled to the radius of each configuration. A linear fit can be made to each equation of state. We fit to the phenomenological relation presented in the previous subsection, equation (17). In Table IV we present the fit parameters $A, B$ for each one of the equations of state studied.

In Fig. 20 we present the damping time of the fundamental mode scaled to the mass of each configuration. In this case, the results can be fitted to the empirical quadratic relation on $M / R$, as presented in Eq. (18). In Table IV we present the fit parameters $a, b$, and $c$.

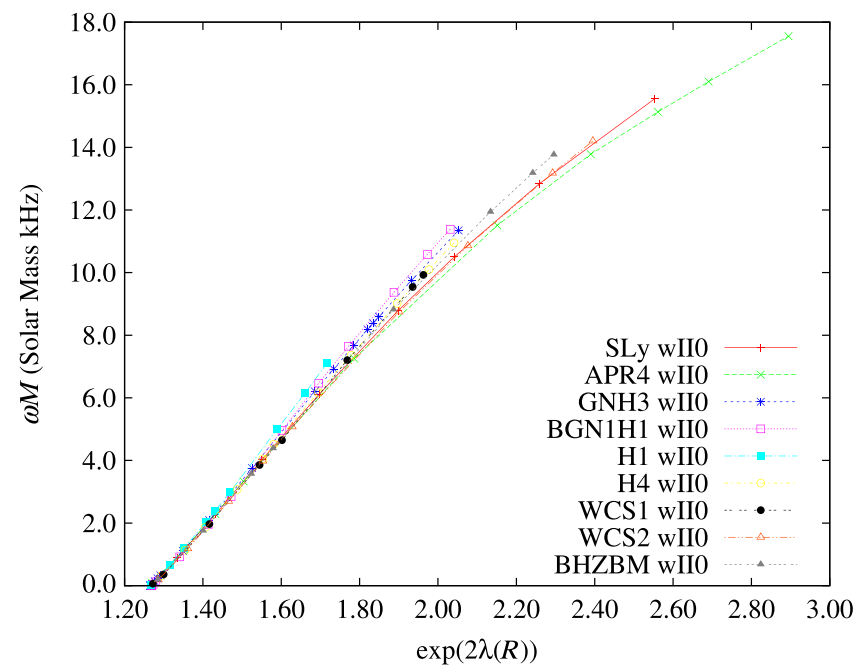

FIG. 12 (color online). Scaled frequency of the fundamental wII mode vs $e^{2 \lambda(R)}$, for the EOS considered in Sec. VA. The frequency vanishes for compactness between $M / R=0.1044$ and $M / R=0.1066$. For low compactness, there are no or almost no hyperons in the core of the star, so at this point all the configurations are basically plain nuclear matter stars.
When we represent the scaled frequency and damping time vs $M / R$, all the wI0 modes have a similar behavior and the coincidence is greater for higher compactness. The only exceptions are WSPHS1-2 EOS (pure quark stars). Their behavior is clearly differentiated from the rest because of the different layer structure found at the exterior of the star.

First excited $w I$ mode.-We present a similar study of the first excited wI mode. In Figs. 21 and 22 we plot the scaled frequency and damping time for the first excited wI mode. The empirical relations (17) and (18), with different parameters can be found in Table V. Note that the scaled frequency and damping time is quite similar for every EOS considered, and the only difference is found for the WSPHS1-2 EOS at low densities, for the same reasons than for the fundamental modes.

Fundamental wII mode.-Finally, in Fig. 23 we plot the frequency of the fundamental wII mode scaled with the mass vs the metric function $e^{2 \lambda(R)}$. As in the previous subsection, it can be seen that there is, for all the equations of state studied, a minimal compactness below which the wII mode vanishes.

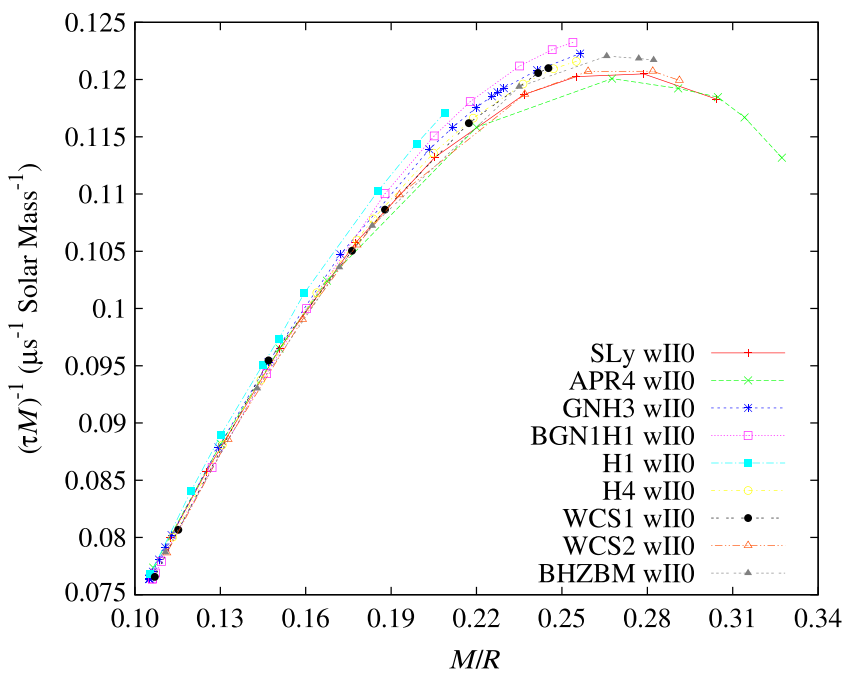

FIG. 13 (color online). Scaled damping time of the fundamental wII mode vs $M / R$, for the EOS considered in Sec. VA. The damping time does not vanish in the limit compactness as the frequency does. 


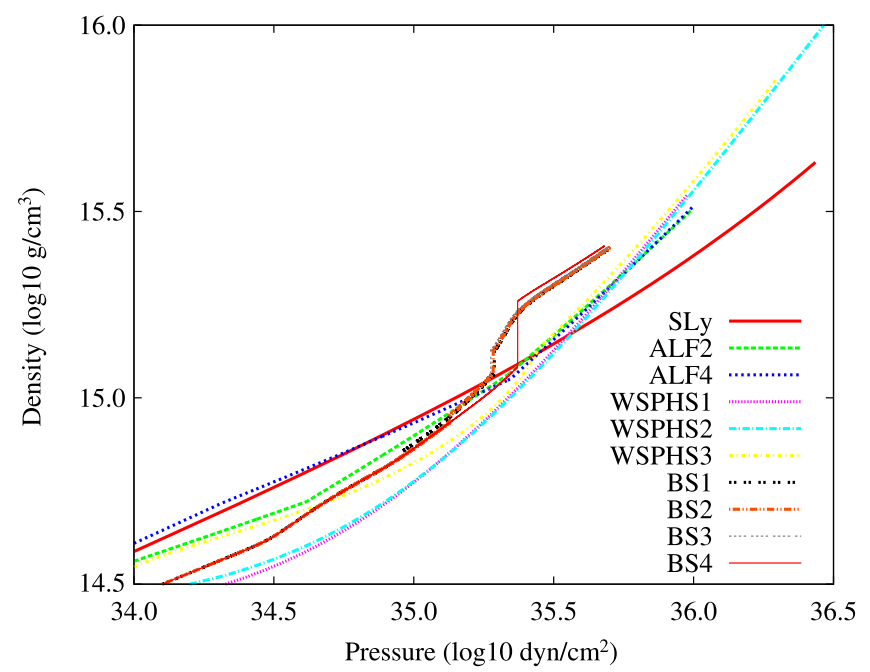

FIG. 14 (color online). Density vs pressure in logarithmic scale in the high density region, for the EOS considered in Sec. V B. Note that hybrid stars present important phase transitions at high pressures.

In Fig. 24 we show the damping time of the wII mode scaled with the mass, where it can be seen that for these limit configurations it tends to a limit $\tau$ dependent of the equation of state. Note that for the hybrid stars considered, the limit configuration is different depending on the matter composition of the outer layers. For hybrid stars ALF2, ALF4 and WSPHS3, the limit configurations have compactness between $M / R=0.1046$ and $M / R=0.1060$, similar to hyperon stars. For BS1-4, the limit configurations have compactness $M / R=0.112$, and for quark stars WSPHS1 and WSPHS2, the limit configurations are quite different and have compactness $M / R=0.126$. These differences are due to the behavior of the different equations

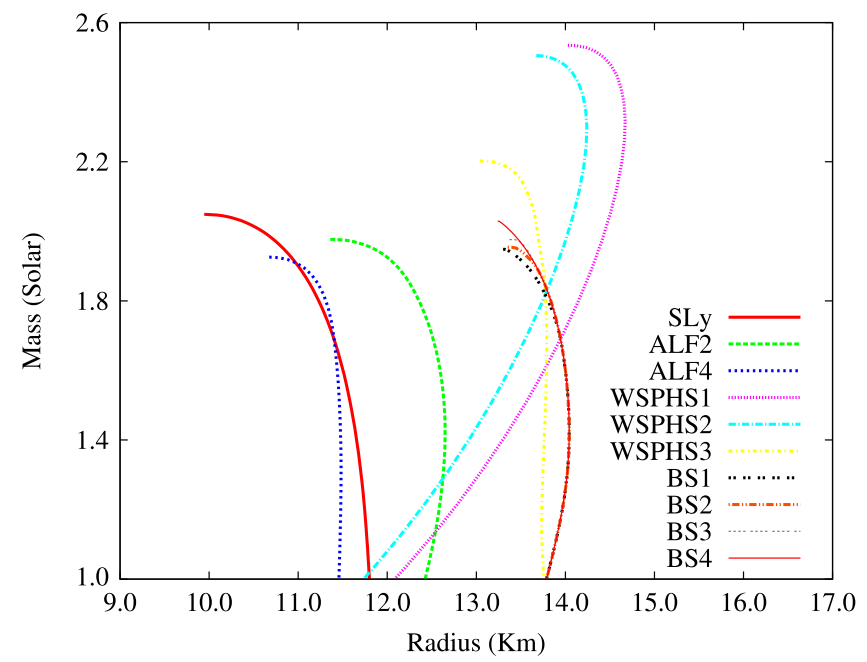

FIG. 15 (color online). Mass vs radius relation for the EOS considered in Sec. VB. All these EOS have maximum mass nearby or beyond $2 M_{\odot}$ and radius beyond $10 \mathrm{Km}$.

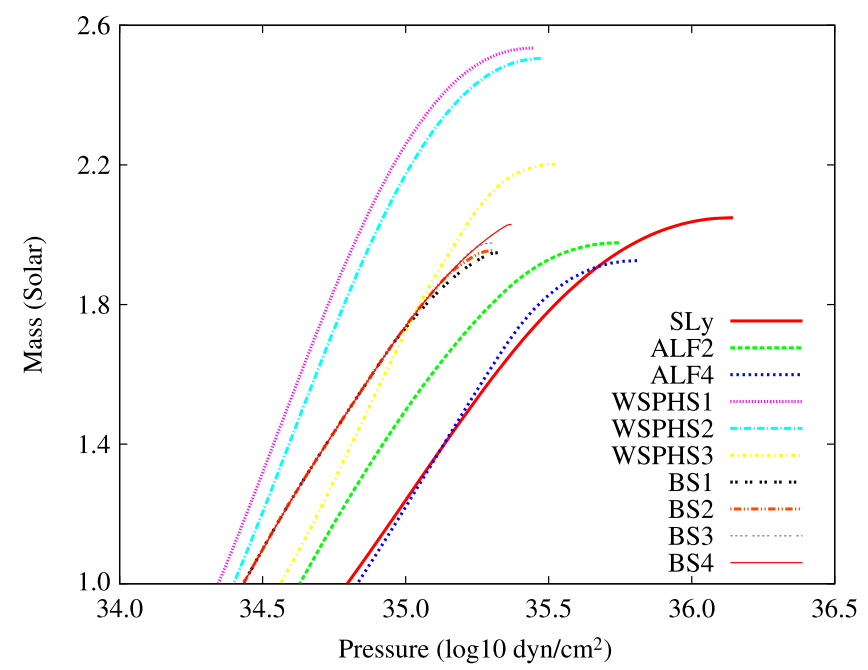

FIG. 16 (color online). Mass vs central pressure relation, for the EOS considered in Sec. V B. In this study we consider only stable configurations between $1 M_{\odot}$ and the maximum mass of the particular EOS.

of state of each configuration at the outer layers of the star. In fact, note that for quark stars WSPHS1 and WSPHS2 the pressure drops to zero with constant density around $2 \times 10^{14} \mathrm{~g} / \mathrm{cm}^{3}$.

\section{BS equations of state}

In order to study the influence of different phases in the compact star, we study four equations of state, considered by Bonanno and Sedrakian with the NL3 parametrization of the Walecka model [19]. These EOS correspond to hybrid stars with hyperons and quark color-superconductivity. They usually have four regions,

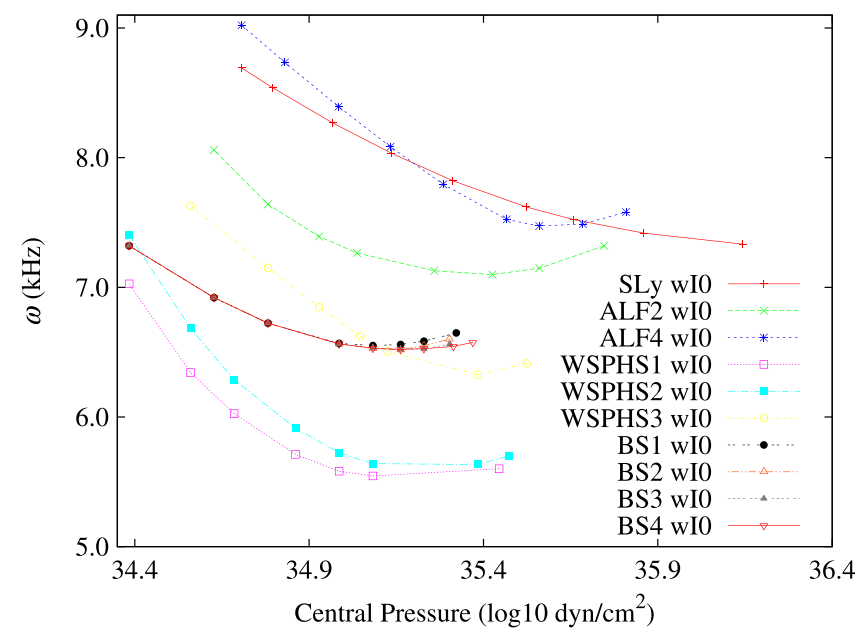

FIG. 17 (color online). Frequency of the fundamental wI mode vs central pressure in logarithmic scale. Hybrid stars tend to have lower frequencies than plain nuclear matter stars or hyperon matter. Pure quark stars have the smallest frequencies, below $6 \mathrm{kHz}$. 


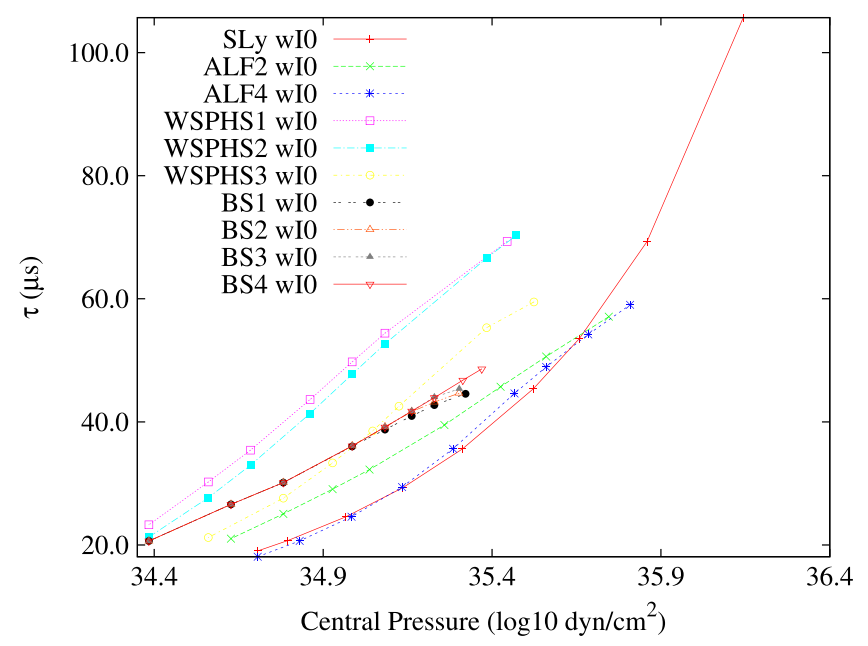

FIG. 18 (color online). Damping time of the fundamental wI mode vs central pressure in logarithmic scale, for the EOS considered in Sec. V B. Hybrid stars are found in between the pure quark matter configurations and the plain nuclear matter ones.

which are, from the inside to the outside: CFL (quark matter three-flavor color-flavor-locked), 2SC (quark matter two-superconducting colors phase), $\mathrm{N}+$ Hyp (nuclear and Hyper-nuclear matter), and $\mathrm{N}$ (nuclear matter). The extent of each region depends on the quark-hadron transition density $\rho_{\text {tr }}$ and the vector coupling constant $G_{V}$. We study four equations of state with $G_{V}=0.6 G_{S}$ and $\rho_{\text {tr }}=2 \rho_{0}$, $3 \rho_{0}, 3.5 \rho_{0}, 4 \rho_{0}$ where $\rho_{0}$ is the density of nuclear saturation.

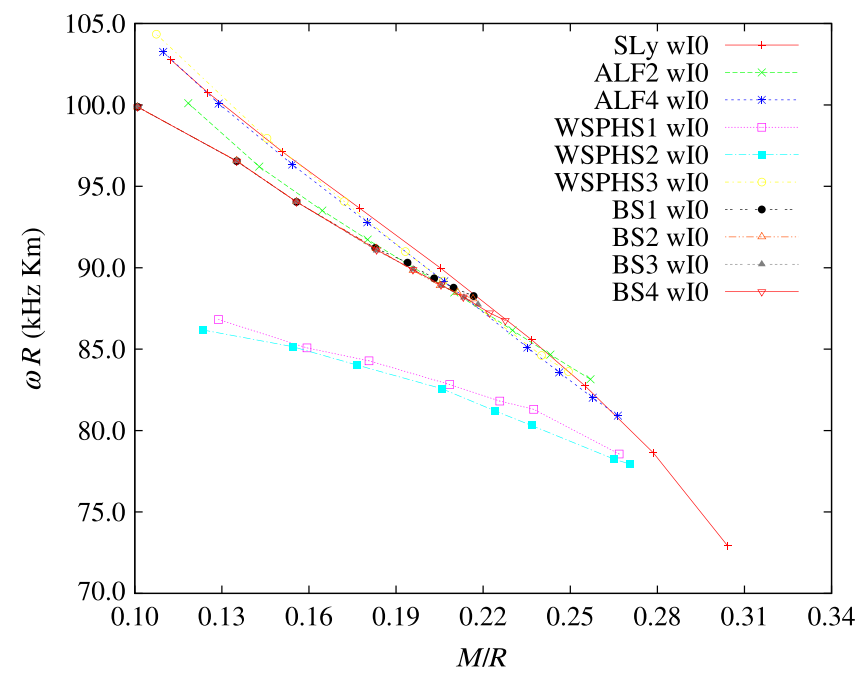

FIG. 19 (color online). Scaled frequency of the fundamental wI mode vs $M / R$, for the EOS considered in Sec. VB. All the scaled frequencies can be fitted to a linear relation with the compactness (17), even the ones for WSPHS1-2. The empirical parameters of hybrid stars are quite different from those of pure quark stars. The fits can be found in Table IV.
In Fig. 25 we plot the equations of state in the high density region. In Fig. 26 we plot the frequency of the fundamental wI mode as a function of the central pressure of the star in logarithmic scale. In Fig. 27 we make a similar plot for the damping time of these configurations.

We can see in Fig. 25 that the BS equations of the state have phase transitions, where for a given pressure, the density suffers a finite jump that increases its value (the EOS become softer). The effect of these phase transitions is reflected clearly in the frequency and damping time of the wI0 modes as it can be seen in Figs. 26 and 27.

\section{EMPIRICAL RELATIONS FOR $W$ MODES}

In Fig. 28 we present $\omega_{I} M$ vs $\omega_{R} M$ for wI0, wI1, and wII0 modes, for all the equations of state studied. This is a plot similar to Fig. 4 of [49] for constant density stars. It can be seen that for the fundamental modes, the scaling with the mass is quite independent of the equation of state.

Now let us define $\bar{\omega}_{R}$ and $\bar{\omega}_{I}$ (real and imaginary part of the $w$ modes in units of the central pressure) by the following relations

$$
\begin{aligned}
\bar{\omega}_{R} & =2 \pi \frac{1}{\sqrt{p_{c}\left(\mathrm{~cm}^{-2}\right)}} \frac{10^{3}}{c} \omega(\mathrm{Khz}), \\
\bar{\omega}_{I} & =\frac{1}{\sqrt{p_{c}\left(\mathrm{~cm}^{-2}\right)}} \frac{10^{6}}{c} \frac{1}{\tau(\mu \mathrm{s})},
\end{aligned}
$$

where $c$ is the speed of light in $\mathrm{cm} / \mathrm{s}$. If we plot $\bar{\omega}_{R}$ vs $\bar{\omega}_{I}$ of wI0, wI1, and wII0 modes for all the equations of state, we obtain Fig. 29 with three curves, one for each type of modes. A remarkable feature is that all the wI0 modes of Fig. 28 can be described by the curve marked wIO in Fig. 29, almost independently of the equation of state. A similar behavior is obtained for wI1 and wII0 modes. One would expect all the wI0 modes of a given EOS to describe a curve depending on one parameter (for instance, the central pressure). And in principle, different equations of state should describe different curves. It is interesting to note that this is not the case, since the empirical relation between $\bar{\omega}_{R}$ and $\bar{\omega}_{I}$ is independent of the equation of state. Hence, all the modes of the same type are located in the same curve in Fig. 29, independently of the EOS. Nevertheless, the parametrization of the curve using the central pressure depends on the EOS. It is interesting to note that the only points that separate slightly from the corresponding line are those of modes for equations of state WSPHS1 and WSPHS2 of quark stars.

This result allows us to present an empirical relation between $\bar{\omega}_{R}$ and $\bar{\omega}_{I}$ that seems to be independent of the equation of the state. As far as we know this has not been obtained before.

For all the EOS except WSPHS1 and WSPHS2, we obtain the following:

For the fundamental wI modes, our results of $\bar{\omega}_{I}$ vs $\bar{\omega}_{R}$ can be fitted to a quadratic relation as follows: 
TABLE IV. Fits for the wI0 modes. Parameters $A$ and $B$ correspond to the linear empirical relation for the frequency (17). Parameters $a, b$, and $c$ correspond to the quadratic empirical relation for the damping time (18).

\begin{tabular}{|c|c|c|c|c|c|c|c|c|c|}
\hline wI0 & ALF2 & ALF4 & WSPHS1 & WSPHS2 & WSPHS3 & BS1 & BS2 & BS3 & BS4 \\
\hline$A$ & $-119.0 \pm 3.0$ & $-141.4 \pm 1.2$ & $-56.5 \pm 3.7$ & $-57.95 \pm 3.1$ & $-145.3 \pm 3.4$ & $-102.1 \pm 1.8$ & $-104.3 \pm 1.7$ & $-104.9 \pm 1.4$ & $-104.7 \pm 1.1$ \\
\hline$B$ & $113.51 \pm 0.59$ & $118.40 \pm 0.25$ & $94.31 \pm 0.75$ & $93.96 \pm 0.65$ & $119.98 \pm 0.66$ & $110.14 \pm 0.32$ & $110.42 \pm 0.31$ & $110.50 \pm 0.25$ & $110.46 \pm 0.20$ \\
\hline$\chi^{2}$ & 0.151 & 0.039 & 0.182 & 0.182 & 0.178 & 0.0359 & 0.0343 & 0.0227 & 0.0174 \\
\hline$a$ & $-1290 \pm 42$ & $-1158 \pm 26$ & $-1319 \pm 75$ & $-295 \pm 35$ & $-1147 \pm 24$ & $-1275 \pm 46$ & $-1263 \pm 46$ & $-1266 \pm 43$ & $-1283 \pm 34$ \\
\hline$b$ & $395 \pm 16$ & $345.3 \pm 9.8$ & $455 \pm 30$ & $445 \pm 14$ & $340.4 \pm 8.7$ & $394 \pm 15$ & $390 \pm 15$ & $391 \pm 14$ & $397 \pm 11$ \\
\hline$c$ & $18.6 \pm 1.4$ & $23.06 \pm 0.88$ & $9.3 \pm 2.3$ & $10.4 \pm 1.4$ & $23.62 \pm 0.76$ & $18.4 \pm 1.1$ & $18.6 \pm 1.1$ & $18.5 \pm 1.1$ & $18.14 \pm 0.90$ \\
\hline$\chi^{2}$ & 0.043 & 0.028 & 0.134 & 0.044 & 0.017 & 0.025 & 0.025 & 0.023 & 0.021 \\
\hline
\end{tabular}

$$
\begin{aligned}
\bar{\omega}_{I}= & (-2.30 \pm 0.13)+(0.58 \pm 0.16) \bar{\omega}_{R} \\
& +(0.0165 \pm 0.0004) \bar{\omega}_{R}^{2},
\end{aligned}
$$

with $\chi^{2}=0.107$.

For the first excited wI mode, we obtain the following fit:

$$
\begin{aligned}
\bar{\omega}_{I}= & (-1.22 \pm 0.13)+(0.485 \pm 0.008) \bar{\omega}_{R} \\
& +(0.00163 \pm 0.00011) \bar{\omega}_{R}^{2},
\end{aligned}
$$

with $\chi^{2}=0.170$. It is almost linear with $\bar{\omega}_{R}$.

For the fundamental wII mode, $\bar{\omega}_{R}$ is quadratic with $\bar{\omega}_{I}$. We obtain the following fit:

$$
\begin{aligned}
\bar{\omega}_{R}= & (4.21 \pm 0.14)+(0.173 \pm 0.012) \bar{\omega}_{I} \\
& +(-0.00524 \pm 0.00020) \bar{\omega}_{I}^{2},
\end{aligned}
$$

with $\chi^{2}=0.165$.

For quark stars (EOS WSPHS1 and WSPHS2) we obtain slightly different values for the parameters of the empirical relation:

For the fundamental wI modes, our results of $\bar{\omega}_{I}$ vs $\bar{\omega}_{R}$ can be fitted to a quadratic relation as follows:

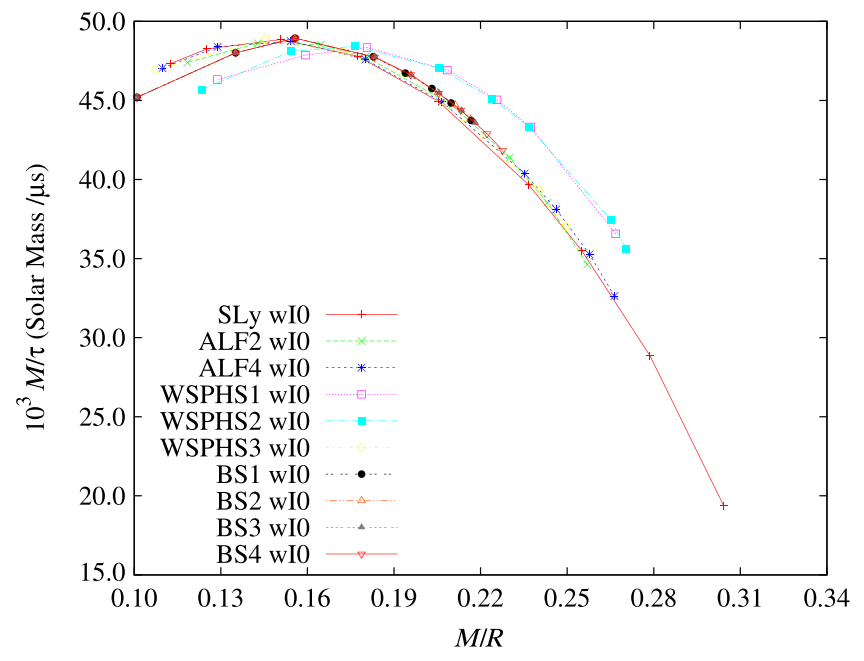

FIG. 20 (color online). Scaled damping time of the fundamental wI mode vs $M / R$, for the EOS considered in Sec. VB. The scaled damping times can be fitted to a quadratic relation with the compactness (18). Again, WSPHS1-2 present a different behavior from the hybrid stars. The fits can be found in Table IV.

$$
\begin{aligned}
\bar{\omega}_{I}= & (-2.20 \pm 0.22)+(0.557 \pm 0.024) \bar{\omega}_{R} \\
& +(0.0148 \pm 0.0006) \bar{\omega}_{R}^{2},
\end{aligned}
$$

with $\chi^{2}=0.0220$.

For the first excited wI mode, we obtain the following fit:

$$
\begin{aligned}
\bar{\omega}_{I}= & (-1.275 \pm 0.097)+(0.476 \pm 0.005) \bar{\omega}_{R} \\
& +(0.0010 \pm 0.00005) \bar{\omega}_{R}^{2},
\end{aligned}
$$

with $\chi^{2}=0.00902$. It is almost linear with $\bar{\omega}_{R}$.

For the fundamental wII mode, $\bar{\omega}_{R}$ is quadratic with $\bar{\omega}_{I}$. We obtain the following fit:

$$
\begin{aligned}
\bar{\omega}_{R}= & (4.32 \pm 0.30)+(0.198 \pm 0.022) \bar{\omega}_{I} \\
& +(-0.0051 \pm 0.0004) \bar{\omega}_{I}^{2},
\end{aligned}
$$

with $\chi^{2}=0.061$.

These relations allow us to obtain $\bar{\omega}_{I}$ if $\bar{\omega}_{R}$ is known. From a practical point of view, these relations can be used to simplify the numerical calculations to obtain quasinormal modes for any equation of state, reducing, in our

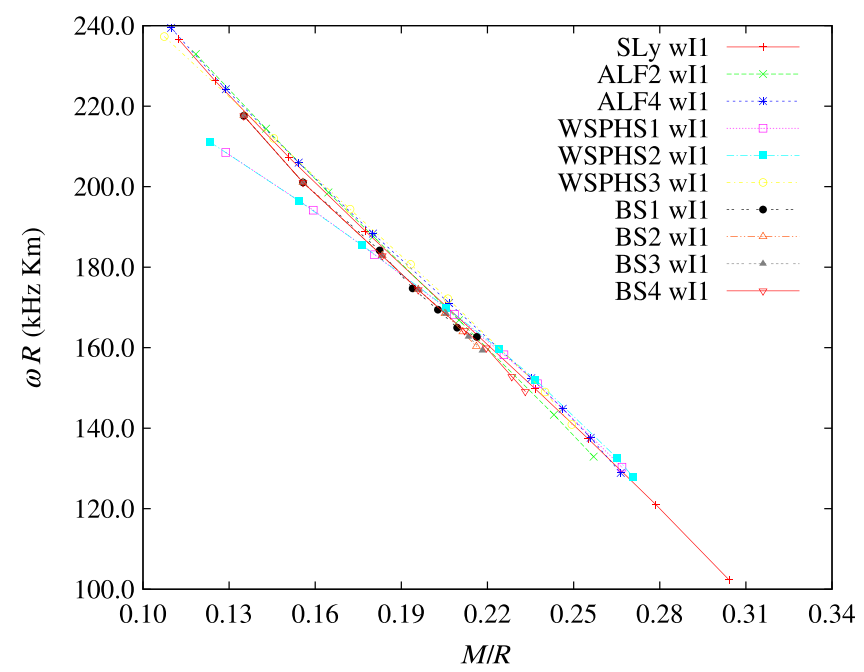

FIG. 21 (color online). Scaled frequency of the first excited wI mode vs $M / R$, for the EOS considered in Sec. V B. In this case, all these EOS fit perfectly the linear relation with the compactness (17). The fits can be found in Table V. 


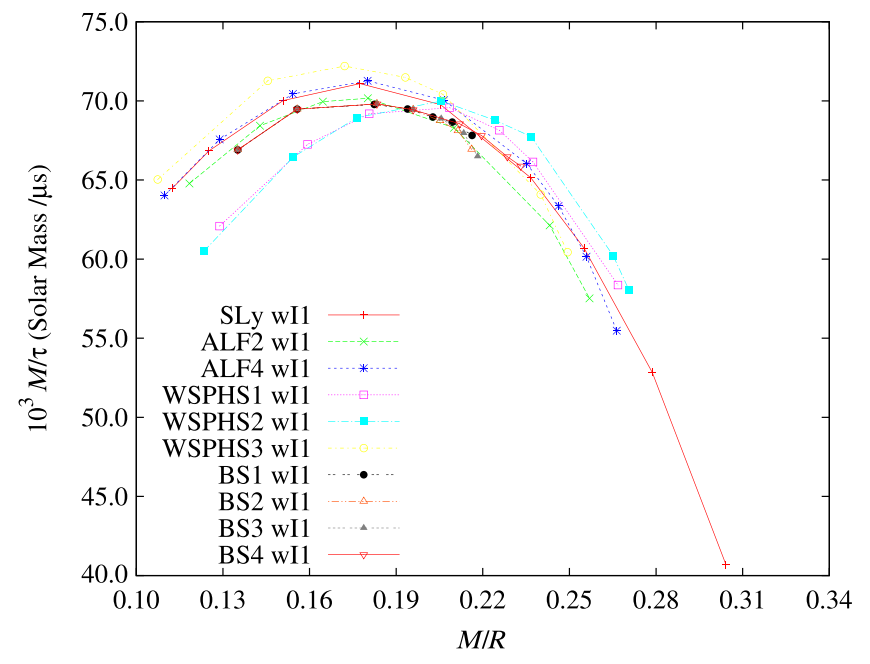

FIG. 22 (color online). Scaled damping time of the first excited wI mode vs $M / R$, for the EOS considered in Sec. VB. A quadratic relation with the compactness (18) can be found for all these EOS. For low compactness WSPHS1-2 configurations the scaled damping time can be a $10 \%$ smaller than for hybrid stars. The fits can be found in Table V.

method, the time necessary to find the zeros of the determinant.

Let us present a possible application of these empirical relations. Suppose that the frequency $\omega(K h z)$ and the damping time $\tau(\mu s)$ of the fundamental mode wI0 of a neutron star are detected. Then, using the Eq. (19) we can plot a line for $\bar{\omega}_{I}$ vs $\bar{\omega}_{R}$ in Fig. 29 with parameter of the line $p_{c}$. The crossing point of this line with the curve marked wI0, given by the empirical relation (20), gives us an estimation of the central pressure $p_{c}$ independently of the equation of state. Now, we can check which equations of state are compatible with this $p_{c}$; i.e., which EOS have the measured wI0 mode near the crossing point for the estimated central pressure. Hence, this method allows us to estimate the central pressure $p_{c}$ and, in some situations, discard certain equations of state. Let us mention that using this $p_{c}$, we can estimate the mass and the radius of the star using our calculations for the possible EOS. Note, that if these data (mass and radius) are measured by other methods, we would have another filter to impose to the EOS.

\section{OTHER RESULTS}

Finally, we study the effect of core-crust transition pressure variations in the quasinormal mode spectrum using our model of crust as a surface energy density enveloping the core. We will consider the SLy equation of state, for which the core-crust transition is found at density $1.29 \times$ $10^{14} \mathrm{~g} / \mathrm{cm}^{3}$. We construct a star of $1.4 M_{\odot}$ with and without a surface energy density enveloping the core of the star at this core-crust transition region. We can compare the difference in the fundamental $w$ modes between our approximation of crust as a thin shell enveloping the core, and the standard calculation. The result is the following: for the fundamental wI mode, the change in the frequency is $0.07 \%$ and the variation in the damping time is $-0.02 \%$. For the fundamental wII mode, the changes are bigger. For the frequency $0.3 \%$ and for the damping time $-0.07 \%$. This indicates that this approximation makes small changes in the $w$ quasinormal spectrum.

Next, we consider this neutron star with $1.4 M_{\odot}$ and surface energy density at the core-crust transition point: $4.8 \times 10^{32} \mathrm{dyn} / \mathrm{cm}^{2}$. Fixing the central pressure, we variate the core-crust transition pressure between $10^{32} \mathrm{dyn} / \mathrm{cm}^{2}$ and $10^{33} \mathrm{dyn} / \mathrm{cm}^{2}$, and compare with the modes for the initial configuration. The impact of transition pressure variations on the frequency is small, between $-0.05 \%$ and $0.08 \%$ for the fundamental wI mode, and between $-0.24 \%$ and $0.35 \%$ for the fundamental wII mode. Similarly, transition pressure variations have a small impact on the damping time, between $0.02 \%$ and $-0.035 \%$ for the fundamental wI mode, and between $0.06 \%$ and $-0.1 \%$ for the fundamental wII mode.

\section{SUMMARY}

In this paper we have studied the axial $w$ quasinormal modes for 18 equations of state. In particular, we consider the influence of the presence of exotic matter in the core of the neutron star.

We have seen that the hyperon core changes the frequency and the damping time of the modes for configurations of high compactness. The frequency increases with the central pressure above $\sim 10^{35} \mathrm{dyn} / \mathrm{cm}^{2}$, where the hyperon phases are more important due to the high density of the star core. This effect is related to the softening of the

TABLE V. Fits for the wI1 modes. Parameters $A$ and $B$ correspond to the linear empirical relation for the frequency (17). Parameters $a, b$, and $c$ correspond to the quadratic empirical relation for the damping time (18).

\begin{tabular}{|c|c|c|c|c|c|c|c|c|c|}
\hline wI1 & ALF2 & ALF4 & WSPHS1 & WSPHS2 & WSPHS3 & BS1 & BS2 & BS3 & BS4 \\
\hline 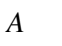 & $-715.8 \pm 4.0$ & $-688.7 \pm 6.8$ & $-560 \pm 19$ & $-562 \pm 18$ & $-672.6 \pm 6.7$ & $-685 \pm 19$ & $-694 \pm 10$ & $-689 \pm 11$ & $-681 \pm 11$ \\
\hline$B$ & $316.98 \pm 0.78$ & $313.5 \pm 1.4$ & $283.0 \pm 4.0$ & $283.3 \pm 3.8$ & $310.0 \pm 1.3$ & $308.9 \pm 3.5$ & $310.4 \pm 2.0$ & $309.7 \pm 2.0$ & $308.3 \pm 2.0$ \\
\hline$x^{2}$ & 0.258 & 1.236 & 5.073 & 6.203 & 0.691 & 1.821 & 0.6037 & 0.662 & 0.921 \\
\hline$a$ & $-1828 \pm 72$ & $-1857 \pm 110$ & $-2026 \pm 103$ & $-2017 \pm 117$ & $-1870 \pm 79$ & $-1536 \pm 142$ & $-1874 \pm 155$ & $-1897 \pm 205$ & $-1458 \pm 85$ \\
\hline$D$ & $637 \pm 27$ & $654 \pm 42$ & $780 \pm 40$ & $785 \pm 47$ & $639 \pm 29$ & $549 \pm 50$ & $661 \pm 55$ & $670 \pm 73$ & $524 \pm 32$ \\
\hline$c$ & $14.7 \pm 2.5$ & $14.2 \pm 3.8$ & $-5.1 \pm 3.9$ & $6.3 \pm 4.5$ & $17.9 \pm 2.5$ & $20.9 \pm 4.3$ & $11.8 \pm 4.7$ & $11.0 \pm 6.3$ & $22.8 \pm 2.9$ \\
\hline$x^{2}$ & 0.1242 & 0.510 & 0.252 & 0.486 & 0.187 & 0.0502 & 0.0584 & 0.1167 & 0.0457 \\
\hline
\end{tabular}




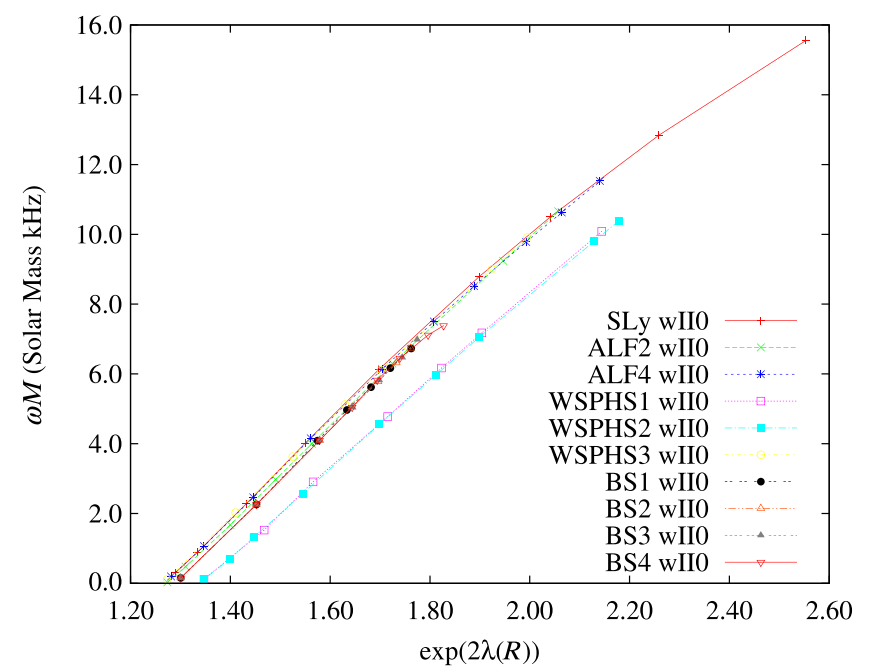

FIG. 23 (color online). Scaled frequency of the fundamental wII mode vs $e^{2 \lambda(R)}$, for the EOS considered in Sec. V B. The frequency vanishes for a compactness that depends on the lower densities of the equation of state considered, around $M / R=$ 0.105 and $M / R=0.110$ for hybrid stars. The most different are the pure quark stars WSPHS1-2, which have the limit at $M / R=$ 0.126 .

equation of state at the inner regions of the star. For stars including quark matter in the core, we obtain a clear influence of the quark presence on the frequency and damping time when represented vs central pressure, with a characteristic behavior for all the EOS with quark matter in the core.

We have obtained phenomenological relations for the frequency and damping time with $M / R$ for wI and wII modes. For some equations of state (for hyperon matter H1

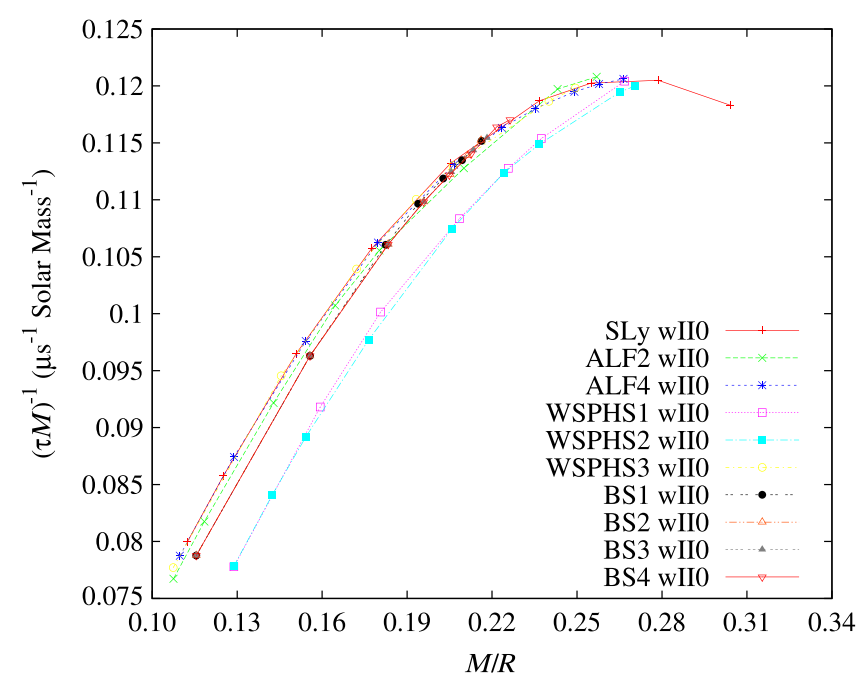

FIG. 24 (color online). Damping time of the fundamental wII mode vs $M / R$, for the EOS considered in Sec. V B. The damping time does not vanish in the limit compactness as the frequency does.

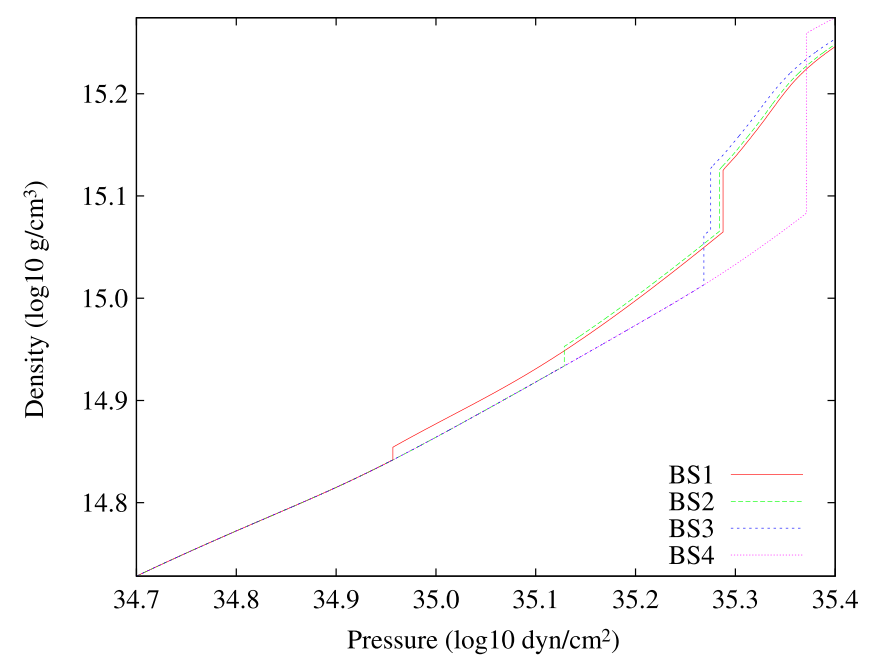

FIG. 25 (color online). Density vs pressure in logarithmic scale for the BS EOS for high densities. Note that at high pressures different phase transitions are taken into account, affecting the stiffness of the EOS.

and BGN1H1, and for pure quark stars WSPHS1-2) the scaling relations are quite different from the rest of the EOS. For the rest of the EOS considered, the scaled relations are quite similar, providing useful information that could be used to measure the radius of a neutron star.

A detailed analysis of four equations of state considered by Bonanno and Sedrakian allows us to study the influence of the presence of different phase transitions in the spectrum of a compact star.

Another interesting result is the new phenomenological relation between the real part $\bar{\omega}_{R}$ and the imaginary part $\bar{\omega}_{I}$ (scaled to the central pressure) of $w$ quasinormal modes,

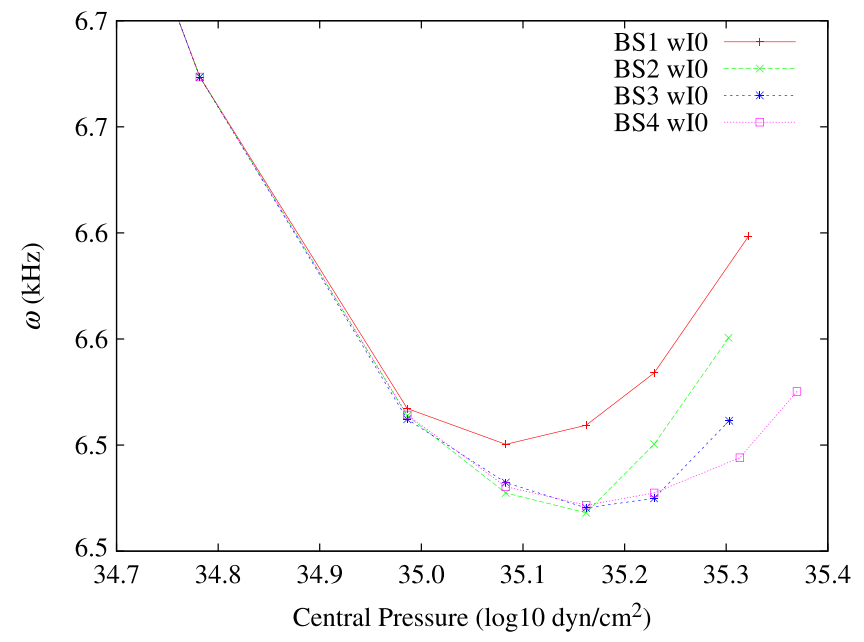

FIG. 26 (color online). Frequency of the fundamental wI mode vs central pressure for the BS equations of state. The phase transitions that can be seen in the previous figure affects the frequency curves. These curves branch once the EOS is affected by the phase transition. 


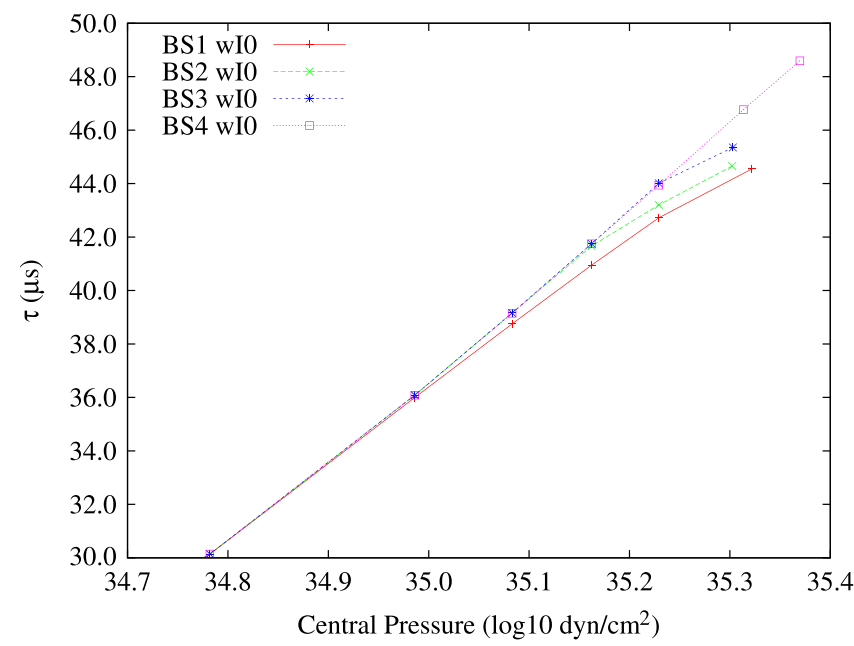

FIG. 27 (color online). Damping time of the fundamental wI mode vs central pressure for the BS equations of state. In a similar way to the frequency, the phase transitions in the equations of state are clearly reflected in the branching of the damping time curves.

valid for all the EOS, except, perhaps, for WSPHS1 and WSPHS2 EOS (quark stars).

The precision of our algorithm allows us to construct explicitly the universal low compactness limiting configuration around $M / R=0.106$ for which the fundamental wII mode vanishes. The existence of this configuration was conjectured by [30].

Finally, we have studied the influence of changes in the core-crust transition pressure obtaining that the influence is very small.

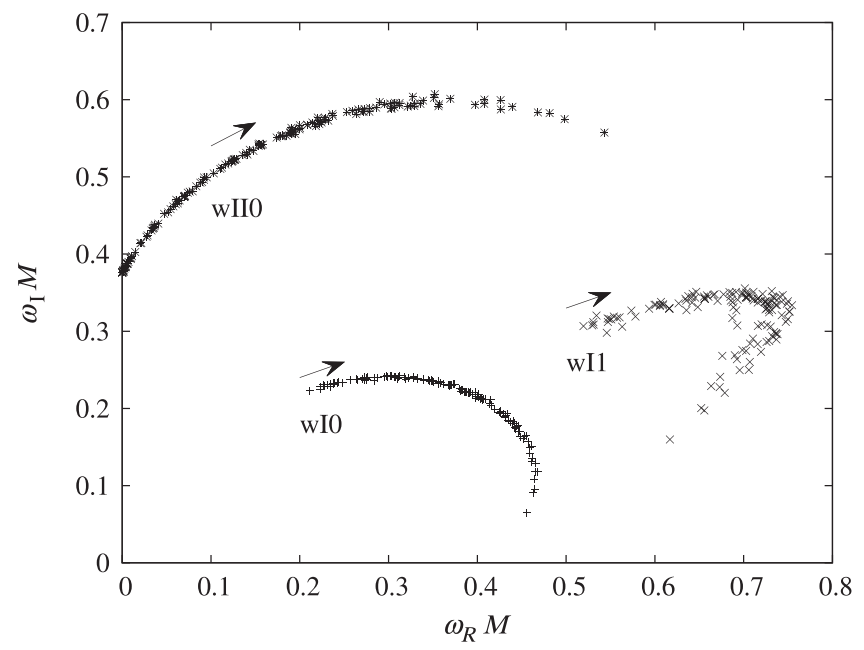

FIG. 28. Adimensional quantities $\omega_{I} M$ vs $\omega_{R} M$ for all $w$ modes and EOS considered. Note that here we choose $c=G=1$, so the products $\omega_{R} M, \omega_{I} M$ are adimensional. It can be seen that the scaling with the mass is quite independent of the equation of state, especially for the fundamental modes. The arrow indicates increasing compactness.

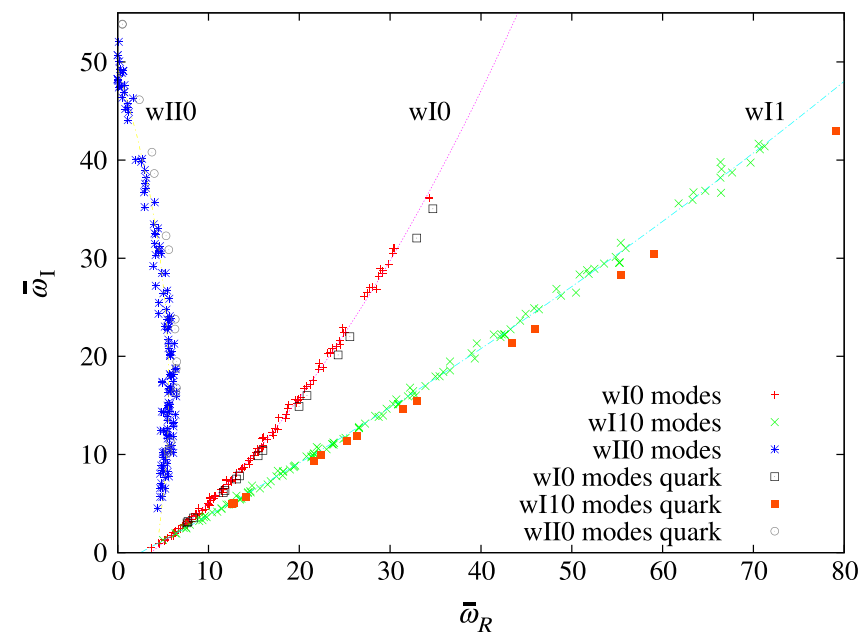

FIG. 29 (color online). $\quad \bar{\omega}_{I}$ vs $\bar{\omega}_{R}$ as defined in Eqs. (19) for all the $w$ modes and for every equation of state we considered in the study. Here it can be seen that the scaling with the central pressure is also quite independent of the equation of state. This universal relation could be used to estimate, for example, the central pressure of the star independently of the EOS. The compactness decreases when increasing $\bar{\omega}_{I}$.

In order to perform this analysis, we developed a new numerical method to calculate $w$-axial quasinormal modes. The use of the phase of the Regge-Wheeler function together with exterior complex scaling, allow us to impose the outgoing wave behavior with stringent conditions. We allow for variable angle in the complex scaling, which makes it possible to obtain new modes and enhances precision with respect to previous works.

We present a complete study of the junction conditions between the exterior and interior solution in a surface of constant pressure (also including the case $p=0$, the border of the star). The matching conditions can be written in terms of a determinant whose zeros correspond to quasinormal modes for the particular integrated configuration.

\section{ACKNOWLEDGMENTS}

We would like to thank I. Bednarek for kindly providing us with the EOS BHZBM, I. Sagert for EOS WSPHS1-3, A. Sedrakian for EOS BS1-4, and S. Weissenborn for EOS WCS1-2. We thank Professor Kostas D. Kokkotas for very helpful discussions concerning quasinormal modes of neutron stars and for the hospitality to J.L.B. We are very grateful to Professor Gabriel A. Galindo for his help concerning the exterior complex scaling method. We also want to thank the referees for his/her very helpful comments and suggestions. This work was supported by the Spanish Ministerio de Ciencia e Innovacion, research Project No. FIS201128013. J. L. B. was supported by the Spanish Universidad Complutense de Madrid. 
[1] M. Pitkin, S. Reid, S. Rowan, and J. Hough, Living Rev. Relativity 14, 5 (2011).

[2] B. Sathyaprakash and B. F. Schutz, Living Rev. Relativity 12, 2 (2009).

[3] N. Andersson, V. Ferrari, D. Jones, K. Kokkotas, B. Krishnan, J. Read, L. Rezzolla, and B. Zink, Gen. Relativ. Gravit. 43, 409 (2011).

[4] N. Andersson and K. D. Kokkotas, Phys. Rev. Lett. 77, 4134 (1996).

[5] M. Shibata, K. Taniguchi, and K. b. o. Uryū, Phys. Rev. D 71, 084021 (2005).

[6] M. Shibata and K. Uryū, Prog. Theor. Phys. 107, 265 (2002).

[7] R. Oechslin, H.-T. Janka, and A. Marek, Astron. Astrophys. 467, 395 (2007).

[8] T. Hinderer, B. D. Lackey, R. N. Lang, and J. S. Read, Phys. Rev. D 81, 123016 (2010).

[9] J.S. Read, C. Markakis, M. Shibata, K.b. o. Uryū, J. D. E. Creighton, and J. L. Friedman, Phys. Rev. D 79, 124033 (2009).

[10] J. Abadie et al. (LIGO Scientific Collaboration), Phys. Rev. D 83, 042001 (2011).

[11] K. D. Kokkotas and B. Schmidt, Living Rev. Relativity 2, 2 (1999).

[12] H.-P. Nollert, Classical Quantum Gravity 16, R159 (1999).

[13] L. Rezzolla, Gravitational Waves from Perturbed Black Holes and Relativistic Stars (ICTP, Trieste, 2003).

[14] A. Haensel, P. Potekhin, and D. Yakovlev, Neutron Stars: Equation of State and Structure, Astrophysics and Space Science Library (Springer, Berlin, 2007).

[15] N. Glendenning, Compact Stars: Nuclear Physics, Particle Physics, and General Relativity, Astronomy and Astrophysics Library (Springer, Berlin, 2000).

[16] H. Heiselberg and M. Hjorth-Jensen, Phys. Rep. 328, 237 (2000).

[17] M. Prakash and J. M. Lattimer, in From Nuclei to Stars, edited by S. Lee (World Scientific, Singapore, 2011), Chap. 12, p. 275.

[18] I. Bednarek, P. Haensel, J. L. Zdunik, M. Bejger, and R. Mánka, Astron. Astrophys. 543, A157 (2012).

[19] L. Bonanno and A. Sedrakian, Astron. Astrophys. 539, A16 (2012).

[20] S. Weissenborn, D. Chatterjee, and J. Schaffner-Bielich, Phys. Rev. C 85, 065802 (2012).

[21] S. Weissenborn, I. Sagert, G. Pagliara, M. Hempel, and J. Schaffner-Bielich, Astrophys. J. Lett. 740, L14 (2011).

[22] J. S. Read, B. D. Lackey, B. J. Owen, and J. L. Friedman, Phys. Rev. D 79, 124032 (2009).

[23] L. Lindblom, Phys. Rev. D 82, 103011 (2010).

[24] O. Benhar, E. Berti, and V. Ferrari, Mon. Not. R. Astron. Soc. 310, 797 (1999).

[25] K. D. Kokkotas, T.A. Apostolatos, and N. Andersson, Mon. Not. R. Astron. Soc. 320, 307 (2001).

[26] O. Benhar, V. Ferrari, and L. Gualtieri, Phys. Rev. D 70, 124015 (2004).

[27] O. Benhar, Mod. Phys. Lett. A 20, 2335 (2005).

[28] V. Ferrari and L. Gualtieri, Gen. Relativ. Gravit. 40, 945 (2008).

[29] D. Chatterjee and D. Bandyopadhyay, Phys. Rev. D 80, 023011 (2009).
[30] D.-H. Wen, B.-A. Li, and P. G. Krastev, Phys. Rev. C 80, 025801 (2009).

[31] T. Regge and J. A. Wheeler, Phys. Rev. 108, 1063 (1957).

[32] F. J. Zerilli, Phys. Rev. Lett. 24, 737 (1970).

[33] K. Thorne and A. Campolattaro, Astrophys. J. 149, 591 (1967).

[34] R. Price and K. Thorne, Astrophys. J. 155, 163 (1969).

[35] K. Thorne, Astrophys. J. 158, 1 (1969).

[36] K. Thorne, Astrophys. J. 158, 997 (1969).

[37] A. Campolattaro and K. Thorne, Astrophys. J. 159, 847 (1970).

[38] L. Lindblom and S. L. Detweiler, Astrophys. J. Suppl. Ser. 53, 73 (1983).

[39] S. Detweiler and L. Lindblom, Astrophys. J. 292, 12 (1985).

[40] S. Chandrasekhar and V. Ferrari, Proc. R. Soc. A 432, 247 (1991).

[41] S. Chandrasekhar, V. Ferrari, and R. Winston, Proc. R. Soc. A 434, 635 (1991).

[42] S. Chandrasekhar and V. Ferrari, Proc. R. Soc. A 434, 449 (1991).

[43] Y. Kojima, Phys. Rev. D 46, 4289 (1992).

[44] N. Andersson, Y. Kojima, and K. D. Kokkotas, Astrophys. J. 462, 855 (1996).

[45] K. D. Kokkotas, Mon. Not. R. Astron. Soc. 268, 1015 (1994).

[46] K. D. Kokkotas and B. F. Schutz, Mon. Not. R. Astron. Soc. 255, 119 (1992).

[47] N. Andersson, K. D. Kokkotas, and B.F. Schutz, Mon. Not. R. Astron. Soc. 274, 9 (1995).

[48] S. Chandrasekhar and S. Detweiler, Proc. R. Soc. A 344, 441 (1975).

[49] N. Andersson, Y. Kojima, and K. D. Kokkotas, Astrophys. J. 462, 855 (1996).

[50] N. Andersson, Proc. R. Soc. A 439, 47 (1992).

[51] L. Samuelsson, N. Andersson, and A. Maniopoulou, Classical Quantum Gravity 24, 4147 (2007).

[52] E. W. Leaver, Proc. R. Soc. A 402, 285 (1985).

[53] M. Leins, H. P. Nollert, and M. H. Soffel, Phys. Rev. D 48, 3467 (1993).

[54] G. Darmois, Mémorial des Sciences Mathématiques (Gauthier-Villars, Paris, 1927), Vol. 25.

[55] A. Lichnerowicz, Théories Relativistes de la Gravitation et de l'Electromagnétism (Maison, Paris, 1955).

[56] S. O'Brien and J. L. Syngr, Comm. Dublin Inst. Adv. Stud. A 9, 1 (1952).

[57] W. Israel, Nuovo Cimento B 44, 1 (1966).

[58] M. Mars and J. M. Senovilla, Classical Quantum Gravity 10, 1865 (1993).

[59] R. Vera, Classical Quantum Gravity 19, 5249 (2002).

[60] M. Mars, Classical Quantum Gravity 22, 3325 (2005).

[61] L. M. González-Romero, Phys. Rev. D 67, 064011 (2003).

[62] M. A. H. MacCallum, M. Mars, and R. Vera, Phys. Rev. D 75, 024017 (2007).

[63] H. Sotani, K. Tominaga, and K.-i. Maeda, Phys. Rev. D 65, 024010 (2001).

[64] C. Misner, K. Thorne, and J. Wheeler, Gravitation, Physics Series (W. H. Freeman, New York, 1973).

[65] U. Ascher, J. Christiansen, and R. D. Russell, Math. Comput. 33, 659 (1979).

[66] J. Aguilar and J. Combes, Commun. Math. Phys. 22, 269 (1971). 
[67] E. Balslev and J. Combes, Commun. Math. Phys. 22, 280 (1971).

[68] B. Simon, Commun. Math. Phys. 27, 1 (1972).

[69] G. Alvarez, R. Damburg, and H. Silverstone, Phys. Rev. A 44, 3060 (1991).

[70] F. Fritsch and R. Carlson, SIAM J. Numer. Anal. 17, 238 (1980).

[71] F. Douchin and P. Haensel, Astron. Astrophys. 380, 151 (2001).

[72] A. Akmal, V.R. Pandharipande, and D. G. Ravenhall, Phys. Rev. C 58, 1804 (1998).
[73] N. Glendenning, Astrophys. J. 293, 470 (1985).

[74] B. D. Lackey, M. Nayyar, and B. J. Owen, Phys. Rev. D 73, 024021 (2006).

[75] S. Balberg and A. Gal, Nucl. Phys. A625, 435 (1997).

[76] M. Alford, M. Braby, M. Paris, and S. Reddy, Astrophys. J. 629, 969 (2005).

[77] N. Andersson and K. D. Kokkotas, Mon. Not. R. Astron. Soc. 299, 1059 (1998).

[78] O. Benhar, E. Berti, and V. Ferrari, Mon. Not. R. Astron. Soc. 310, 797 (1999). 